\title{
Open Access bei der Veröffentlichung rechtswissenschaftlicher Fachliteratur - was soll das?
}

\author{
Hanjo Hamann \& Daniel Hürlimann*
}

A. Einleitung $\ldots \ldots \ldots \ldots \ldots \ldots \ldots \ldots \ldots \ldots \ldots, \quad 4$

B. Thesen........................... 6

I. Rechtswissenschaft interessiert nur JuristInnen...................... 6

II. Deutsches Jura interessiert nur Deutsche.

III. Juristische Karrieren kommen ohne Metriken aus.

IV. JuristInnen haben keine Peer Review.
V. Rechtswissenschaft ist drittmittelfern.

VI. Rechtswissenschaft ist papierbasiert und IT-fern....................... 18

VII. Verlage müssen marktgerechte Preise setzen ....................... 19

VIII. Juristische Verlagspreise sind angemessen. .......................... 22

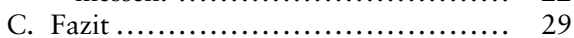

Die deutschsprachige Rechtswissenschaft ist eine der Fachdisziplinen mit der geringsten Durchsetzungsrate von Open Access überhaupt. Woher kommt diese Zurückhaltung gegenüber einer in anderen Wissenschaften enthusiastisch vorangetriebenen und von der Politik breit geförderten Bewegung? Will man der Rechtswissenschaft keine anachronistische Fortschrittsverweigerung unterstellen, sollten sich sachliche Gründe ausmachen lassen, warum sie dem Phänomen Open Access mit Skepsis begegnet, oder jedenfalls keine eigenen Anstrengungen in diese Richtung unternimmt. Solche Gründe werden in der Rechtswissenschaft bisher kaum öffentlich kommuniziert und allenfalls im informellen Kreis erörtert. Der vorliegende Beitrag sichtet die Gründe und leitet aus der Perspektive eines advocatus diaboli acht Thesen her, warum die institutionellen Rabmenbedingungen der Rechtswissenschaft mit Open Access unvereinbar sein könnten. Eine argumentative Auseinandersetzung mit diesen Thesen verspricht einen differenzierteren Diskurs, der die Rechtswissenschaft in ibren Eigenheiten ernst nimmt, ohne dabei die Potentiale von Open Access zu verleugnen.

* Dr. Dr. Hanjo Hamann ist Senior Research Fellow am Max-Planck-Institut zur Erforschung von Gemeinschaftsgütern (Bonn) und Mitherausgeber von JLL (www.LanguageAndLaw.eu) und Rechts|Empirie (www.RechtsEmpirie.de), Ass.-Prof. Dr. Daniel Hürlimann ist Assistenzprofessor für Informationsrecht an der Universität St.Gallen und Herausgeber von sui generis (www.sui-generis.ch). Die Autoren danken (nicht nur, aber insbesondere) Alexander Peukert für die gemeinschaftliche Ausrichtung der Tagung „Open Access für die Rechtswissenschaft: Pflicht oder Privatsache?“ im Oktober 2018, auf der dieses Sonderheft beruht (www.jurOA.de\#2018), Johannes Rux für die unermüdliche, umsichtige und wohlwollende Redaktion der Beiträge, Christian Wolf und der Universitätsbibliothek Marburg für wertvolle Einblicke und die geduldige Beantwortung allzu neugieriger Fragen, sowie Ralf Schimmer und der Max Planck Digital Library (MPDL) für die Möglichkeit, das Sonderheft Open Access zu veröffentlichen. 


\section{A. Einleitung}

Open Access, also der freie - technisch unbeschränkt, rechtlich nachnutzbar und kostenfrei angebotene ${ }^{1}-$ Zugang zu wissenschaftlichen Publikationen, ist in den meisten Fachdisziplinen weiter fortgeschritten als in der Rechtswissenschaft. In Untersuchungen zum Anteil von Open Access in verschiedenen Disziplinen schneidet die Rechtswissenschaft, sofern sie überhaupt als eigene Disziplin berücksichtigt wird, ${ }^{2}$ in der Regel unterdurchschnittlich ab: Studien, die den Anteil an OA-Publikationen in der Rechtswissenschaft gesondert ausweisen, beziffern diesen mit fünf bis zehn Prozent. ${ }^{3}$ In fast allen anderen Disziplinen sind die OA-Anteile bedeutend höher, Vorreiter ist die Biomedizin mit einem Open-Access-Anteil von etwa 60 Prozent. ${ }^{4}$ Wo die Rechtswissenschaft zusammen mit den Geisteswissenschaften und teilweise sogar den Kunstwissenschaften erfasst wird, ${ }^{5}$ erreicht sie OA-Anteile bis zu 32 Prozent. ${ }^{6}$ Die benachbarten Sozialwissenschaften dagegen kommen in manchen Untersuchungen auf einen fast doppelt so hohen OA-Anteil. ${ }^{7}$

Dabei dürften die meisten Studien den OA-Anteil in der Rechtswissenschaft sogar noch deutlich überschätzen, denn viele beruhen auf der Auswertung von Datenbanken und Plattformen, in denen tendenziell mehr digital als gedruckt veröffentlichte rechtswissenschaftliche Beiträge erfasst sind. Dazu gehören die Datenbank Web of Science ebenso wie die Suchmaschine Google Scholar oder auch die Zitations- und Abstractsammlung Scopus. Soweit ersichtlich, existiert im deutschsprachigen Raum nur eine einzige belastbare Untersuchung zum Verfügbarkeitsgrad rechtswissenschaftlicher Beiträge; danach sind in der Schweiz höchstens 19 Prozent

1 Näher zur Definition H. Hamann, in diesem Heft, S. 100 ff.

2 Sie fehlt in V. Larivière / C. R. Sugimoto, Do authors comply when funders enforce open access to research?, Nature 2018, S. 483 (perma.cc/J4TG-Y2CZ); H. Piwowar / J. Priem / V. Larivière / J. P. Alperin / L. Matthias / B. Norlander / A. Farley / J. West / S. Haustein, The state of OA: a large-scale analysis of the prevalence and impact of Open Access articles, PeerJ 6:e4375 (perma.cc/743NQHHG); J. Bosman / B. Kramer, Open access levels: a quantitative exploration using Web of Science and oaDOI data, PeerJ Preprints 6:e3520v 1 (perma.cc/6QMC-Z6SL); Science-Metrix, Open access availability of scientific publications, Final Report, January 2018 (perma.cc/F7ZG-C32D).

3 C. Haijem/S. Harnad/Y. Gingras, Ten-Year Cross-Disciplinary Comparison of the Growth of Open Access and How it Increases Research Citation Impact, IEEE Data Engineering Bulletin 2005/4 (perma.cc/6PRD-U45W), S. 39 (42); Europäische Kommission, Open science monitor, Trends for open access to publications, Percentage of open access publications (gold and green) by FOS (fields of science and technology), Law: $90.9 \%$ Not OA.

4 Piwowar, Open Access (Fn. 2), S. 14.

5 Überblick bei A. Severin / M. Egger / M.P. Eve / D. Hürlimann, Discipline-specific open access publishing practices and barriers to change: an evidence-based review, F1000Research 2018, 7:1925 (perma.cc/9DBY-Z2G6).

6 A. Martín-Martín/R. Costas/T. v. Leeuwen/E. Delgado López-Cózar, Evidence of Open Access of scientific publications in Google Scholar: a large-scale analysis, Journal of Informetrics 2018 (perma.cc) K9F9-ZMGT), S. 819 (831).

$761 \%$ bei H. R. Jamali/M. Nabavi, Open access and sources of full-text articles in Google Scholar in different subject fields, Scientometrics 2015, S. 1635; nur 17 \% dagegen bei Bosman/Kramer (Fn. 2). 
der Beiträge aus rechtswissenschaftlichen Zeitschriften kostenlos (nicht zwangsläufig Open Access) zugänglich. ${ }^{8}$

Diese ungewöhnlich geringe Durchsetzungsrate von Open Access führt zur Frage nach dem Warum. Sie wird kaum je expliziert und allenfalls im vertraulichen Gespräch von Open-Access-AktivistInnen als anachronistischer Sonderweg einer Disziplin abgetan, die ohnehin an vielen Fronten mit ihrer Wissenschaftlichkeit hadert. ${ }^{9}$ Der vorliegende Beitrag dreht den Spieß um. Er lädt zu einem Gedankenexperiment ein, welches das übliche Narrativ vom Kopf auf die Füße stellt: Statt die Rechtswissenschaft zu belehren, warum sie sich der Open-Access-Bewegung anschließen sollte, fragt der vorliegende Beitrag, warum sie es bislang wohl nicht getan hat. Worin könnte ihre Skepsis begründet liegen? Warum bringen JuristInnen gegenüber Open Access so wenig Enthusiasmus auf? ${ }^{10}$

Inspiriert in Titel und Thesenformat durch einen provokanten Zwischenruf von Kostorz vor einigen Jahren, ${ }^{11}$ soll der vorliegende Beitrag die Hindernisse zusammentragen, die nach Beobachtung der Autoren in informellen Gesprächen gegen Open Access in der deutsch(sprachig)en Rechtswissenschaft ins Feld geführt werden. Sich daran argumentativ abzuarbeiten, verspricht eine differenziertere Auseinandersetzung, die besser auf bisherige Einwände zu entgegnen hilft und die juristische Fachdisziplin in ihren Eigenheiten ernst nimmt, ohne zugleich neue Ansätze abzulehnen. Aus der Perspektive eines advocatus diaboli greifen die Autoren ${ }^{12}$ deshalb acht Thesen zu Open-Access-hinderlichen Rahmenbedingungen der Rechts-

8 B. Dengg, Bestehende Infrastrukturen für juristische Forschung und deren Kosten, Vortrag Bern 27.5.2016 (perma.cc/ZRU2-HFKN), S. 23. Die Untersuchung basiert auf einer Stichprobe von 9 rechtswissenschaftlichen Zeitschriften aus der Schweiz (AJP, forumpoenale, sic!, successio, ZBJV, SJZ, ZBI, SZZP und jusletter) und berücksichtigt Publikationen im Zeitraum 2010-2015.

9 Ganz schlaglichtartig aus dem neueren Schrifttum etwa diverse AutorInnen, Perspektiven der Rechtswissenschaft in Deutschland, JZ-Themenheft 14/2013, S.693-723; D. Basak / M. Reiß / R. Schimmel, Wissenschaftlichkeit der Rechtswissenschaft? Überlegungen zum Umgang mit Plagiaten in rechtswissenschaftlichen Publikationen und Prüfungsarbeiten, RW 2014, S. 277; A. Dyevre, The Future of Legal Theory and the Law School of the Future, Antwerpen 2015; R. van Gestel / H.-W. Micklitz / E. L. Rubin (Hrsg.), Rethinking Legal Scholarship. A Transatlantic Dialogue, Cambridge 2017 (mit mehreren Beiträgen aus dem deutschen Raum).

10 Aufbauend auf früheren Gedanken von H. Hamann, Open Access in der Rechtswissenschaft, GRUR 2016, S. 1140 (1142 l. Sp.), Abschnitt „Woher rührt die OA-Skepsis von Juristen?“ (hanjo.1hamann.de/research/grur2016-1140.pdf) und ders., Freier Zugang zur juristischen Fachliteratur im Spiegel der Open-Science-Bewegung, sui generis 3 (2016), S. 96 (103), Abschnitt „Woher der Widerstand von Juristen gegen Open Access? “ (Rn. 24) (sui-generis.ch/28).

11 P. Kostorz, Review-Verfahren bei der Veröffentlichung juristischer Fachartikel - Was soll das?, KJ 2016, S. 417.

12 Der Beitrag wurde gemeinschaftlich bearbeitet, aber die Entwurfsverantwortung für Thesen 1, 2, 5 und 6 lag beim Autor Hürlimann, die Entwurfsverantwortung für Thesen 3, 4, 7 und 8 beim Autor Hamann. 
wissenschaft auf, ${ }^{13}$ die sodann auf ihre Tragfähigkeit kritisch hinterfragen werden. Diese Thesen lauten in etwas provokanten Zuspitzungen wie folgt:

1. Rechtswissenschaft interessiert nur JuristInnen.

2. Deutsches Jura interessiert nur Deutsche.

3. Juristische Karrieren kommen ohne Metriken aus.

4. JuristInnen haben kein Peer Review.

5. Rechtswissenschaft ist drittmittelfern.

6. Rechtswissenschaft ist papierbasiert und IT-fern.

7. Verlage müssen marktgerechte Preise setzen.

8. Juristische Verlagspreise sind angemessen.

Einige dieser Thesen betreffen das von JuristInnen wahrgenommene „Proprium“ ihrer Disziplin, ${ }^{14}$ also ihr akademisches Selbstverständnis. Eine kritische Nabelschau, inwieweit dieses Selbstverständnis mit Open Access vereinbar ist oder dadurch umgekehrt in Frage gestellt wird, erscheint auch deshalb besonders lohnenswert.

\section{B. Thesen}

\section{Rechtswissenschaft interessiert nur JuristInnen.}

Ein erstes Hindernis könnte aufmerksamkeitsökonomischer Art sein: Während die Forschungsergebnisse anderer Disziplinen, zum Beispiel der Medizin, für ein breites Publikum interessant und zum Teil lebenswichtig sind, könnte Rechtswissenschaft als Binnendiskurs einer spezialisierten Fachgemeinschaft schlicht keinen Bedarf nach öffentlicher Kommunikation von Wissenschaftsergebnissen haben. JuristInnen, so die Prämisse, haben ohnehin alle einen Zugang zu juris und Beck Online oder Swisslex - und wer ihn nicht hat, will ohnehin nicht am Diskurs teilnehmen.

Die Erfahrung mit juristischen Open-Access-Publikationen zeigt indes, dass sowohl Medien als auch die Öffentlichkeit durchaus an den Erkenntnissen der Rechtswissenschaft interessiert sind. Auch wenn in Zeitungen und im Fernsehen unter dem Stichwort „Wissenschaft“ meistens Aktualitäten aus der Medizin oder den Naturwissenschaften präsentiert werden, interessieren sich Medien durchaus auch für das Recht - wie die Justizberichterstattung und die öffentliche Berichterstattung über laufende Gesetzgebungsprojekte belegen.

13 Darunter werden hier nur Aufsätze, Monographien, Sammelbände und Kommentierungen von Rechtswissenschaftlern für Rechtswissenschaftler verstanden; die Praktikerliteratur mag Open Access noch andere, u.U. erhöhte Widerstände entgegensetzen, die hier ausgeblendet bleiben.

14 Vgl. Ch. Engel / W. Schön, Das Proprium der Rechtswissenschaft, Tübingen 2007, S. IX: „Juristen stellen ihre Texte nicht im Internet zur Verfügung. Juristische Aufsätze sind nicht peer reviewed. Juristen achten nicht auf den impact factor. Juristen finanzieren ihre Forschung nicht aus Drittmitteln. Juristen haben keine Sonderforschungsbereiche.". 
Auch rechtswissenschaftliche Fragestellungen sind für eine breitere Öffentlichkeit von Interesse, wie die Social-Media-Resonanz von Open-Access-Zeitschriften belegt. So verfügt der Twitter-Account des German Law Journal über 2.200 Follower, derjenige der Schweizer sui generis über 700. ${ }^{15}$ Darunter sind zahlreiche JournalistInnen, sodass sogar die in vergleichsweise jungen Onlinezeitschriften wie sui generis $^{16}$ publizierten Beiträge ihren AutorInnen Medienanfragen und Interviews einbringen, in Zeitungen ${ }^{17}$ ebenso wie im Radio ${ }^{18}$ und Fernsehen. ${ }^{19}$

Neben JournalistInnen sind auch WissenschaftlerInnen anderer Disziplinen mitunter auf rechtswissenschaftliche Literatur angewiesen. In einer Zeit zunehmend interdisziplinärer Forschungsfragen haben auch ForscherInnen anderer Disziplinen eine berechtigte Erwartung, juristisches Fachwissen abrufen zu können. Da geht es NichtjuristInnen genauso wie JuristInnen, die auf Erkenntnisse aus anderen Wissenschaften angewiesen sind. Wer beispielsweise im Gesundheitsrecht forscht, muss regelmäßig auf Publikationen aus der Medizin und der Ethik zurückgreifen. Derzeit ist dies nur mit unverhältnismäßig hohem Aufwand oder durch sogenannte Schattenbibliotheken möglich. In der Schweiz ist die Nutzung der Schattenbibliothek Sci-Hub aufgrund der Eigengebrauchsregelung rechtmäßig, ${ }^{20}$ in Deutschland hingegen bestehen diesbezüglich unterschiedliche Auffassungen. ${ }^{21}$ Solche juristischen Graubereiche bei der Nachnutzung steuerfinanzierter Wissenschaftserkenntnisse können sich für die betroffenen WissenschaftlerInnen karriereschädlich und für die Gesellschaft innovationshemmend auswirken.

Es gibt zweifellos Publikationen, die in erster Linie für ExpertInnen auf dem jeweiligen Gebiet von Interesse sind. Die vermeintlichen Hürden sind aber oft weniger hoch als gedacht. Dies zeigt sich unter anderem an den Zugriffszahlen auf Fachzeitschriften, die vor der Umstellung auf Open Access in einer Auflage von 300 Ex-

15 Siehe twitter.com/ger_law_journal sowie twitter.com/suigeneris_ch (Stand am 4. August 2019).

16 Gegr. 2004 unter sui-generis.ch durch den Beitragsautor Hürlimann.

17 Interview mit Sarah Progin-Theuerkauf in der SonntagsZeitung vom 7. September 2014 im Nachgang zur ihrer Publikation: S. Progin-Theuerkauf, Asylrechtliche Überlegungen zur Schutzbedürftigkeit Edward Snowdens, sui-generis 2014, S. 22 (perma.cc/J84W-NWFQ). Darauf bezogen auch Artikel in der Wochenzeitung: Die Schweiz müsste Snowden aufnehmen (perma.cc/WY57-MUV8); Artikel auf heise online: Gutachten: Snowden könnte in der Schweiz auf freies Geleit hoffen (perma.cc/ PJ7H-8B8V); Artikel in 20 Minuten: Kommt Edward Snowden bald in die Schweiz? (perma.cc/ Z4PV-98M7).

18 Interview mit Thomas Burri (perma.cc/37WT-3D5R) im Nachgang zu seiner Publikation: T. Burri, Verfassungslos: Verfassungs- und staatsrechtliche Bedenken anlässlich des Bundesgerichtsentscheides zur Bündner Spracheninitiative, sui-generis 2017, S. 148 (perma.cc/SV6X-JXU9).

19 Studiogespräch mit Charlotte Sieber-Gasser (perma.cc/ANR2-YMC8) im Nachgang zu ihrer Publikation: C. Sieber-Gasser, Das Ende der bilateralen Handelsabkommen?, sui-generis 2015, S. 1 (perma.cc/VK88-T5GK).

20 Swiss Academies of Arts and Sciences, Open Science in Switzerland: Opportunities and Challenges, Bern 2019 (perma.cc/9BMT-4HAY), S. 2.

21 E. W. Steinhauer, Die Nutzung einer 'Schattenbibliothek' im Licht des Urheberrechts, Library Ideas 2016 (perma.cc/MVX4-VA6R), S. 128 (132). 
emplaren erschienen sind und nach der Umstellung mehrere Zehntausend Zugriffe pro Monat verzeichnen konnten. ${ }^{22}$

Schließlich lassen sich ohne Schwierigkeiten Rechtsgebiete ausmachen, an denen auch juristische Laien ein sachlich begründetes Interesse haben, wie nicht zuletzt das Arbeits- oder Mietrecht. Deshalb wird zumindest in der Schweiz vermehrt gefordert, dass auch Gesetzeskommentare frei zugänglich sein sollten. So meinte der Forschungsratspräsident des Schweizerischen Nationalfonds (SNF) in einem Radio-Interview: „In der Rechtswissenschaft werden Kommentare über Gesetze publiziert, zum Beispiel der Zürcher Kommentar oder der Berner Kommentar. Es ist sehr wichtig für die Gesellschaft, dass die Interpretation der Gesetze wissenschaftlich aufgearbeitet wird, um ein Verständnis zu erhalten, was ein Gesetz bedeutet. Und diese Bücher, die müssen unbedingt öffentlich zugänglich sein. "23 Auch der Schweizer Staatssekretär für Bildung, Forschung und Innovation äußerte sich dahin, dass Gesetzeskommentare in Zukunft frei zugänglich sein sollen. ${ }^{24}$ Diese Aussagen zeigen, dass Rechtswissenschaft wohl doch nicht nur JuristInnen interessiert.

\section{Deutsches Jura interessiert nur Deutsche.}

Wenn Rechtswissenschaft nicht nur JuristInnen interessiert, trifft vielleicht eine etwas zurückhaltendere Reformulierung dieser These zu: Selbst wenn die Öffentlichkeit sich für rechtswissenschaftliche Erkenntnisse interessiert, wird sich für deutsche Rechtswissenschaft ja wohl allenfalls die deutsche Öffentlichkeit interessieren. Dann wären die bisher etablierten Verbreitungswege durch öffentliche Bibliotheken in allen größeren Städten ja womöglich ausreichend, um den Informationsbedarf der Öffentlichkeit zu decken, ohne dass es einer verstärkten Publikation gleich im weltumspannenden Internet bedürfte.

Die Rechtswissenschaft unterscheidet sich in der Tat von anderen Disziplinen unter anderem auch durch ihre nationale Grundausrichtung. Trotz der wachsenden Bedeutung internationalen, insbesondere europäischen, Rechts, finden sich die meisten Gesetze - und damit die meisten wissenschaftlichen Desiderate - noch immer auf der Ebene (und in der Sprache) der Nationalstaaten. Schon deshalb wird in den Rechtswissenschaften weltweit überwiegend in der jeweiligen Landessprache publi-

22 So berichtet von Marin Dacos im Video "Privés de savoir? » (youtube.com/watch?v=WnxqoP-c0ZE - Aussage bei 6:16: "En base d'une revue qui avait 300 abonnés papiers à des revues qui ont 20'000, 50'000, parfois 80'000 visites dans un seul mois. »); in Deutschland wiesen Chr. Asschenfeldt / R. Honekamp, Freier Zugriff auf wissenschaftliche Beiträge?, ZRP 2004, S. 247 darauf hin, „dass die intelligentesten und zündensten Ideen nicht notwendig ihren Ursprung in der Akademie haben.".

23 Interview mit Matthias Egger, Forschungsratspräsident des Schweizerischen Nationalfonds, Aussage bei 11:00 (perma.cc/B92J-XS5Q).

24 M. Dell'Ambrogio, Der Bund und Open Access (tube.switch.ch/videos/cd8ad92f), Aussage bei 08:04: «Les éditeurs sur le domaine du droit se concentrent sur les Lehrbücher et les commentaires deviennent du libre accès. » 
ziert. Die meisten anderen Wissenschaftsdisziplinen hingegen suchen sich universalere Gegenstände und sind deshalb auch in ihren institutionellen Grundstrukturen viel stärker internationalisiert. Die Gesetze der Naturwissenschaft sind im Unterschied zu jenen des Rechts auf der ganzen Welt identisch. Deshalb ist es naheliegend, dass in diesen Disziplinen auf Englisch publiziert wird. In der Rechtswissenschaft hingegen finden sich sogar unterschiedliche Jurisdiktionen innerhalb desselben Sprachraums, weshalb das Interesse an Publikationen aus anderen Jurisdiktionen gering erscheinen mag.

Dagegen spricht indes die folgende Beobachtung: Auch wenn aktuelle Rechtsfragen nicht in allen deutschsprachigen Ländern gleich zu beantworten sind, lassen sich doch thematische Konjunkturen erkennen, die in sehr ähnlich gelagerten Verfassungsordnungen zwangsläufig Übertragungen nahelegen. Als Beispiel sei auf die Frage verwiesen, ob eine Open-Access-Veröffentlichungspflicht für Dissertationen eingeführt werden kann. Diese Diskussion läuft aktuell in Deutschland, ${ }^{25}$ befeuert durch den Rechtsstreit um die Satzung der Universität Konstanz, ${ }^{26}$ ebenso wie in Österreich ${ }^{27}$ und der Schweiz. ${ }^{28}$ Auch wenn sich die Gesetze in Deutschland, Österreich und der Schweiz unterscheiden, gibt es viele Überschneidungen: Die Wissenschaftsfreiheit wird in allen drei Ländern verfassungsrechtlich garantiert, ${ }^{29}$ die Argumente und Überlegungen sind häufig die gleichen. Ähnliches gilt etwa für die Gesetzgebungslehre (Legistik), die in der Schweiz viel stärker ausgebaut ist als in Deutschland, aber hierzulande aufmerksam verfolgt und seit Jahrzehnten wissenschaftlich begleitet wird. ${ }^{30}$ Je einfacher der Zugang zur Forschungsliteratur der jeweils anderen Jurisdiktion, desto schneller die Diffusion von Erkenntnissen und desto geringer das Risiko, das Rad doppelt zu erfinden.

Deutsches Recht interessiert aber nicht einmal nur die deutschsprachige Öffentlichkeit. Aufgrund der starken Stellung Deutschlands innerhalb der Europäischen Union sind rechtswissenschaftliche Erkenntnisse aus Deutschland in allen EU-Mitgliedstaaten nachgefragt, wie sich etwa an der mittlerweile stark europäischen Ausrichtung der als German Law Journal bezeichneten Open-Access-Zeitschrift

25 E. Steinhauer, in diesem Heft, S. $31 \mathrm{ff}$.

26 Dazu das deutsch-schweizerische Autorenduo H. Hamann/F. Graf, Müssen Wissenschaftler ihre Ergebnisse frei zugänglich machen?, LTO v. 27.9.2017 (lto.de/persistent/a_id/24747).

27 E. Staudegger, Open-Access-Veröffentlichungspflicht für Dissertationen?, ALJ 2018, S. 1 (perma.cc/ F87D-8W5F).

28 A. Kley, Freie Bahn für Open Access, Plädoyer 2018, S. 36 (perma.cc/9W3B-P6F9).

29 Art. 5 Abs. 3 des deutschen Grundgesetzes, Art. 17 des österreichischen Staatsgrundgesetzes, Art. 20 der Schweizer Bundesverfassung.

30 Dazu ausf. H. Hamann, Aktuelle Entwicklungen in der Wissenschaft von der Gesetzestechnik www.LegistiK.de, Zeitschrift für Gesetzgebung 4/2019, im Ersch., gestützt nicht zuletzt auf Berichte zum Stand der Legistik in Deutschland, Österreich und der Schweiz, die in der maßgeblichen OpenAccess-Zeitschrift publiziert wurden (U. Karpen, H. Schäffer bzw. L. Mader, jeweils in LeGes 17 [2006] Ausg. 2, unter leges.weblaw.ch/legesissues/2006/2.html). 
ablesen lässt. Darüber hinaus haben zahlreiche Länder deutsches Recht - insbesondere Zivilrecht - übernommen, sodass RechtshistorikerInnen das enorme Interesse vor Augen steht, das der deutschen Pandektistik ${ }^{31}$ heute in Asien entgegengebracht wird. Ebensolche Wertschätzung gilt auch der neueren Wissenschaftsliteratur: Juristische Wissenschaftsverlage aus Deutschland erzielen im (überwiegend asiatischen) Ausland inzwischen ähnlich hohe Umsätze wie im deutschen Heimatmarkt. ${ }^{32}$ Auch das schweizerische Zivilgesetzbuch war Gegenstand internationaler Übernahmen oder Transplantate, etwa in das türkische Recht. ${ }^{33}$ Dementsprechend international ist das Interesse an der wissenschaftlichen Auseinandersetzung mit deutschsprachigen Rechtsordnungen. Es erscheint in Deutschland kaum eine Festschrift, in der nicht ausländische BeiträgerInnen Substanzielles zum deutschen Recht oder seine anhaltenden Auswirkungen auf ihre jeweiligen Heimatrechtsordnungen vorbringen.

In diesem Kontext zeigt sich, dass Open-Access-Publikationen nicht nur den $\mathrm{Zu}$ gang zur Rechtsliteratur verbessern, sondern auch ihre Zugänglichkeit: Sie ermöglichen auch der türkischen Privatrechtsprofessorin ohne Deutschkenntnisse, den deutschsprachigen Artikel einer ZGB-Professorin aus der Schweiz zur Kenntnis zu nehmen, weil Übersetzungsprogramme zwar nicht immer einwandfreie Ergebnisse liefern, aber kontinuierlich zuverlässiger werden und schon heute Texte aus völlig fremden Sprachen erschließen helfen. Beispielsweise war einer der Autoren für eine Konferenz im deutschsprachigen Teil Italiens mit dem kürzlich in Kraft getretenen italienischen Patientenverfügungsgesetz befasst, ${ }^{34}$ und konnte dank des maschinell angelernten Übersetzungsdienstes www.DeepL.com den Gesetzestext auf erstaunlich hohem sprachlichen Niveau erschließen.

Mithin ist also auch die reformulierte These, wonach deutsche Rechtswissenschaft nur für deutschsprachige Rezipienten Relevanz entfaltet, im Ergebnis zweifelhaft. Deutsches Recht und deutsche Rechtswissenschaft interessieren auch ÖsterreicherInnen und SchweizerInnen, JuristInnen in anderen EU-Staaten, in Klein- und Ostasien, und vielen anderen Jurisdiktionen, von denen wir noch gar nicht viel gehört haben können, weil es ihnen bisher an Zugang zur und Zugänglichkeit der deutschen Rechtsliteratur ermangelte.

31 Pandektistik ist die auf den Pandekten (von Justinian zusammengestellte Auszüge aus den Schriften der klassischen römischen Juristen) aufbauende Rechtswissenschaft des 19. Jahrhunderts; vgl. dazu den Eintrag zu Pandektistik auf Rechtslexikon.net (perma.cc/BU4V-VRR2).

32 Vgl. H. Hamann, Referendarsstation im juristischen Wissenschaftsverlag Mohr Siebeck, JuS 3/2015, S. 32 (33): „Fast die Hälfte seines Umsatzes macht das Unternehmen im Ausland“ (hanjo.1hamann.de/research/jus2015-3_32.pdf); weiter denken Asschenfeldt/Honekamp, Zugriff (Fn. 22), S. 247: „Open Access würde Übersetzungen fördern“.

33 P. Breitschmid/A. Tugrul, 100 Jahre Schweizerisches ZGB, 80 Jahre Türkisches ZGB - Konvergenzen und Divergenzen, Berlin 2008.

34 Legge 22 dicembre 2017, n. 219, Norme in materia di consenso informato e di disposizioni anticipate di trattamento (perma.cc/KBU3-DCSM). 


\section{Juristische Karrieren kommen ohne Metriken aus.}

In den meisten Fachdisziplinen beruhen akademische Karriereentscheidungen auf Metriken: Die Berufung, Bewertung und Beförderung von WissenschaftlerInnen erfolgen aufgrund von Zitationsberechnungen und Einflussmaßen (z.B. Impact Factor oder Hirsch-Index), weshalb die Sichtbarkeit und Zitationshäufigkeit wissenschaftlicher Beiträge maßgeblich über den Verlauf wissenschaftlicher Karrieren bestimmen. Vor diesem Hintergrund wird ein wesentlicher Vorteil von Open-AccessPublikationen darin gesehen, dass sie dank einfacher Onlineverfügbarkeit mehr LeserInnen und intensivere Rezeption erhalten. Da die deutschsprachige Rechtswissenschaft solche Metriken nicht kennt oder darauf verzichtet, ${ }^{35}$ bedarf sie auch keiner aufmerksamkeitsheischenden Onlinepublikationen, so die dritte hier zu untersuchende These.

In der Tat lässt sich der sogenannte „Open Access Citation Advantage“ empirisch belegen: In einer bis 2015 durchgeführten kontinuierlichen Literatursichtung stellten 46 von 70 durchgeführten Studien (66 \%) eine größere Zitationshäufigkeit von Open-Access- im Vergleich zu konventionellen Druckpublikationen fest; nur 17 Studien $(24 \%)$ belegten keinen solchen Vorteil - aber auch nicht unbedingt einen Nachteil. ${ }^{36}$ Eine der 46 Studien, die den Zitationsvorteil dokumentierten, wird auch in der juristischen Literatur mitunter rezipiert. ${ }^{37}$ Eine weitere hat hierzulande bislang kaum Echo erzeugt, scheint aber die einzige zu sein, die sich spezifisch rechtswissenschaftlichen Texten zuwendet: Sie belegt, dass US-amerikanische RechtswissenschaftlerInnen im Durchschnitt 60,2 \% häufiger Beiträge aus offenen als aus geschlossenen Fachzeitschriften (law reviews) zitieren (innerhalb einer Schwankungsbreite je nach Zeitschrift von 17 bis $90 \%$ ) und dass auch Gerichte noch um 41,4 \% häufiger Open-Access-Beiträge zitieren. ${ }^{38}$ Doch selbst im angloamerikanischen Rechtsraum, wo es immerhin vor allen anderen Disziplinen (schon

35 Für Deutschland vgl. K. Purnhagen / N. Petersen, Evaluation of Academic Legal Publications in Germany, in R. van Gestel / A. Lienhard (Hrsg.), Evaluating Academic Legal Research in Europe, 2019; für die Schweiz ausf. M. Schmied / K. Byland / A. Lienhard, Procedures and criteria for evaluating academic legal publications: Results of a survey in Switzerland, Research Evaluation 27 (2018), S. 335 (doi.org/10.1093/reseval/rvy020).

36 Vgl. SPARC Europe, The Open Access Citation Advantage Service (OACA), Stand 2016 ("In 2016 SPARC Europe decided not to further update The Open Access Citation Advantage Service since the citation advantage evidence has now become far more common knowledge to our authors."), abgerufen am 21.1.2019 unter www.sparceurope.org/what-we-do/open-access/sparc-europe-open-accessresources/open-access-citation-advantage-service-oaca.

37 Vgl. M. Schweizer, Warum diese Dissertation nicht in einem Verlag erschienen ist, 2005 („,Der freie Zugang hat auch für die Autoren messbare Vorteile“), abgerufen am 21.1.2019 unter www.markschweizer.ch/?publications=kognitive-tauschungen-vor-gericht; A. Abegg, Offene Wissenschaft Erste Erfahrungen mit ‘open access`- Publikationsplattformen im Bereich der Sozial- und Geisteswissenschaften, Jusletter 1.9.2008, S. 4 vor Fn. 18 („weit grösserer Streuungsbereich von <open access>-Publikationen infolge des freien Zugangs“); beide m.Verw. auf S. Lawrence, Free online availability substantially increases a paper's impact, Nature 411 (2001), S. 521.

38 J.M. Donovan / C.A. Watson / C. Osborne, The Open Access Advantage for American Law Reviews, Edison Law + Technology (Journal of the Patent \& Trademark Office Society) 2015-03A mit 
im 18. Jahrhundert) „Juristen waren, die sich erstmals dafür interessierten, wer von wem wie oft zitiert wird“, bleibt die bibliometrische Einflussmessung eine Kuriosität für LiebhaberInnen; in der deutschen Rechtswissenschaft ist sie noch „gar nicht angekommen“. 39

Selbst wenn man allerdings einen Zitationsvorteil für die deutsche Rechtswissenschaft einmal unterstellt, ließe sich immerhin einwenden, dass juristische Karriereentscheidungen doch nach wie vor auf kommissionsbasierten Leseverfahren und dem subjektiv empfundenen und informell kommunizierten Renommeé bestimmter Verlage und Zeitschriften beruhen. ${ }^{40} \mathrm{Da}$ Open-Access-Zeitschriften bislang durchweg jüngeren Datums sind und deshalb wenig Reputation vorweisen können, ließe sich daraus schließen, dass ihr Vorteil in puncto Publikumsreichweite ohne Wirkung bliebe.

Doch es gibt gegenläufige Anzeichen: In einer quantitativen Erhebung der meistzitierten deutschen Rechtszeitschriften finden sich unter den vier meistzitierten Zeitschriften des Strafrechts zwei reine Onlinezeitschriften (ZIS und HRRS), ${ }^{41}$ die trotz ihres jungen Alters (6 bzw. 12 Jahre zum Zeitpunkt der Erhebung) nicht nur breite Wahrnehmung, sondern auch hohe Wertschätzung unter StrafrechtswissenschaftlerInnen genießen. ${ }^{42}$ Solche Wertschätzung resultiert natürlich großteils aus dem Ruf einer professionellen, fachlich kompetenten Schriftleitung - mindestens ebenso aber darauf, dass die Schriftleitung gute Bedingungen für eine strenge Qualitätsauslese vorfindet: Je größer die Nachfrage nach einer Zeitschrift und je flexibler ihre Heftplanung, desto selektiver kann die Schriftleitung agieren. Open Access kann dabei sowohl durch größere Reichweite und kürzere Publikationszeiten die Attraktivität für AutorInnen und damit das Manuskriptangebot erhöhen, ${ }^{43}$ als auch auf rigide Heftstrukturen verzichten, die zu fixen Terminen „gefüllt“ werden müssen. Damit können es sich Open-Access-SchriftleiterInnen eher leisten, Beiträge abzulehnen, oder zumindest in weitere Überarbeitungsrunden zu verschieben, als SchriftleiterInnen konventioneller Papierzeitschriften. Umgekehrt sind sie auch flexibler darin, Beiträge zu tagesaktuellen Themen zeitnah zu veröffentlichen, können

dem Fazit: “The open access advantage is real, sizable, and consistent.”, verfügbar unter www.jptos.org/news/375.

39 H. Hamann, Die Fußnote, das unbekannte Wesen - Potential und Grenzen juristischer Zitationsanalyse, RW 2014, S. 501 (502, 504).

40 Einzige Erhebung zur wahrgenommenen „qualitativen Bedeutung“ juristischer Zeitschriften: $M$. Gröls/T. Gröls, Ein Ranking juristischer Fachzeitschriften, JZ 17/2oo9, Beil. S. 40, archiviert unter web.archive.org/20150428171538/mohr.de/fileadmin/user_upload/Zeitschriften/JZ/Ranking.pdf.

41 Hamann, Zitationsanalyse (Fn. 39), S. 523.

42 Das Inhaltsverzeichnis der Anthologie zum zehnten Jubiläum der ZIS (Zeitschrift für Internationale Strafrechtsdogmatik) liest sich wie ein Who is Who deutscher Strafrechtswissenschaft: T. Rotsch (Hrsg.), Zehn Jahre ZIS - Zeitschrift für Internationale Strafrechtsdogmatik, Baden-Baden 2018; vgl. auch ders., in diesem Heft, S. 124 ff.

43 Im Vergleich zu konventionellen Zeitschriften gleichen Gründungsdatums. 
also die in manchen Papierzeitschriften in Monaten oder gar Jahren bemessene Wartezeit bis zum Abdruck erheblich unterbieten. All diese Aspekte spielen wohl eine Rolle in den Reputationsdynamiken des Onlinepublizierens, ${ }^{44}$ ganz abgesehen von dem Gemeinplatz, dass auch Autoren, deren Leistung nicht in Metriken gemessen wird, „gern“ gelesen und zitiert werden. ${ }^{45}$

Angesichts dieser Überlegungen erscheint es wahrscheinlich, dass sich bestimmte Open-Access-Zeitschriften in naher Zukunft ein Ansehen erarbeitet haben werden, das für die Karrierewege der dort Publizierenden förderlich ist, ${ }^{46}$ ohne dass es einer zwischengeschalteten Metrisierung überhaupt noch bedarf. Der dokumentierte Reichweitenvorteil von Open-Access-Zeitschriften wird sich mittelfristig also auch in juristischen Evaluierungs- und Berufungsverfahren bemerkbar machen.

\section{JuristInnen haben keine Peer Review.}

Das Publikationswesen vieler Fachdisziplinen, so eine verbreitete Wahrnehmung, schließt ehrenamtlich engagierte WissenschaftlerInnen in Wertschöpfungsketten zusammen, deren staatlich finanziertes Ergebnis schließlich an private ProfiteurInnen verschenkt wird: Steuerfinanzierte Forschung in naturwissenschaftlichen Disziplinen entsteht, indem staatlich angestellte WissenschaftlerInnen ihre Erkenntnisse mithilfe automatisierten Computersatzes (insb. LaTeX) dokumentieren, an eine aus der staatlichen Wissenschaft rekrutierte Schriftleitung weiterreichen, die sie durch andere staatliche WissenschaftlerInnen begutachten lässt (peer review) und zuletzt an einen börsennotierten Großverlag abgibt, der zu minimalen Kosten im asiatischen Ausland sehr teure Dateien herstellt. Da wurde schnell die Frage nach dem Mehrwert von Wissenschaftsverlagen laut. ${ }^{47}$ In der Rechtswissenschaft hingegen beteiligen sich WissenschaftlerInnen bei Zeitschriftenaufsätzen fast gar nicht an den Satzarbeiten, werden fast nie zu deren Begutachtung herangezogen, ${ }^{48}$ und er-

44 Vgl. T. Rotsch, Fünf Jahre ZIS, ZIS 2011, S. 285 f. zum Ziel, die Zeitschrift „jedem Interessierten unproblematisch und weltweit zugänglich zu machen“ und der resultierenden „freundlichen Aufnahme der ZIS in Wissenschaft und Praxis“.

45 Für das Habilitationswesen bezeichnend bspw. G. Wagner/B. Dauner-Lieb, Forschung im Selbstportrait, AcP 216 (2016), S. 325: „Im Rahmen des herkömmlichen juristischen Rezensionswesens ist es unmöglich“, den „Werken junger Zivilrechtswissenschaftler“ „die Verbreitung und Anerkennung, die sie verdient haben“, zuteil werden zu lassen; die dort ebenfalls festgestellte „Bereitschaft zur Teilnahme“ von Habilitierten belegt deren Interesse an Publikumsreichweite.

46 Sei es unmittelbar durch Reputationsgewinn oder mittelbar durch den Selektionsdruck höherer Qualitätsansprüche der Zeitschrift.

47 L.M. Hilty, Was leisten Wissenschaftsverlage heute eigentlich noch?, Informatik Spektrum 38 (2015), S. 302-305 (www.netzpolitik.org/2015/was-lei); zur „Wertschöpfungskette“ schon Th. Pflüger/D. Ertmann, E-Publishing und Open Access - Konsequenzen für das Urheberrecht im Hochschulbereich, ZUM 2004, S. 436 (439).

48 Anders bspw. die vorliegende Zeitschrift; vehement ablehnend dagegen Kostorz, Review-Verfahren (Fn. 11); M. A. Niggli, Zur Kritik des aktuellen Zustandes der Wissenschaft, ContraLegem 2018, S. 4 (8); als "marginal criticism" werten dies K. Purnhagen / N. Petersen, Evaluation of Academic Legal Publications in Germany, in R. van Gestel / A. Lienhard (Hrsg.), Evaluating Academic Legal Research in Europe, im Erscheinen 2019, unter 8. - und weiter: "less controversial is the introduc- 
halten stattdessen (zumindest in Deutschland) sogar noch Tantiemen - als wohl „eine der letzten Disziplinen [...], deren Autoren für Aufsätze vergütet werden “. 49 Wohl deshalb, so unsere vierte These, hat sich in der Rechtwissenschaft kein solcher Leidensdruck entwickelt, ${ }^{50}$ wie er in anderen Disziplinen zur Flucht vor Großverlagen und zur Gründung digitaler Repositorien (z.B. ArXiv.org) und Megajournals (z.B. PLOS ONE) führte.

Gleichwohl stellt sich auch für juristische Wissenschaftsverlage die Frage nach dem Mehrwert von Verlagsdienstleistungen. Die unbestreitbaren Annehmlichkeiten einer vom Verlag übernommenen Satz- und Herstellungsarbeit - zumindest im Falle von Fachzeitschriften - stehen einer in vielen Fällen eher rudimentären Qualitätsauslese gegenüber, die oft weder nach Aufwand noch nach Selektivität dem Peer-Review-Verfahren anderer Disziplinen nahekommt. ${ }^{51}$ Entsprechend laut war schon vor Jahrzehnten die Klage über eine nicht abebbende Flut neuer - nicht immer besonders anspruchsvoller - Zeitschriften, mit denen Fachverlage sich ihre eigene Nachfrage schaffen. ${ }^{52}$ Auch Qualifikationsschriften und Monographien werden weithin auf Grundlage akademischer Voten und eines von etwaigen ReihenherausgeberInnen autonom bestimmten und vom Verlag allenfalls am Rande begleiteten Auswahlverfahrens veröffentlicht und mit sehr überschaubarem wirtschaftlichen Risiko steuerfinanziert hergestellt. ${ }^{53}$

Auch die angedeutete Wertschöpfungskette bedarf natürlich eines organisierten Rahmens, durch den Verlage einen infrastrukturellen Mehrwert für die Wissenschaft schaffen (durch Ansprache von HerausgeberInnen, Organisation von Herausgebersitzungen, Nachschau der Rezensionen zu veröffentlichten Werke, etc.). Solche Infrastrukturen werden allerdings durch digitale Direktkommunikation, kollaborative Publikationswerkzeuge (etwa OJS und OMP, d.h. Open Journal Sys-

tion of more peer-review elements. Double-blind peer reviews for publications would probably increase the quality of the acceptance process.".

49 Hamann, Open Access (Fn. 10); ders., Freier Zugang (Fn. 10).

50 Für rechtsökonomische Zeitschriften allerdings Ch. Engel, Give the Journals Back to the Scientists, JITE 2004, S. 35: "Originally, the main service was physical distribution. But with the advent of the Internet, demand for this service is fading away. [...] typically the only valuable good provided by the publishing houses is electronic distribution.”.

51 Damit ist nicht gesagt, dass Peer Review zwangsläufig die bessere Qualitätsauslese darstellt; jedenfalls aber die aufwändigere - das scheint uns im Kontext des Mehrwerts entscheidend. Ähnl. sogar V. Haug, Open Access in Baden-Württemberg: Rechtswidriger Zweitveröffentlichungszwang zwischen Urheber- und Hochschulrecht, OdW 2019, S. 89 (90) (perma.cc/7CU5-SWVM).

52 M. Peltzer, Eine neue juristische Fachzeitschrift erscheint, JZ 1983, S. 77, zit. nach Hamann/Idler (Hrsg.), Zeitgeistreiches, Tübingen 2015, S. $131 \mathrm{f}$.

53 Bei Qualifikationsschriften meist in einer Mischkalkulation aus Druckkostenzuschüssen, die von AutorInnen oder ihren (meist öffentlichen) Zuschussgebern finanziert werden, und Handelspreisen in solcher Höhe, dass sich die Herstellungs- und Vertriebskosten schon nach Belieferung der juristischen Fachbibliotheken amortisiert haben. Dazu schon R. M. Hilty, Das Urheberrecht und der Wissenschaftler, GRUR Int 2006, S. 179 (185): „In der Realität gehen Verlage [...] oft - wiewohl bereichsabhängig und länderspezifisch - keine Risiken ein.“ 
tems und Open Monograph Press) und öffentliche Fachrepositorien mit fortgeschrittenen Archivierungs- und Indizierungsfunktionen (etwa LawArXiv oder $<$ intR>2Dok) zunehmend günstiger, teils sogar ganz verzichtbar. ${ }^{54}$ Damit sinkt der komparative Mehrwert gegenwärtiger Verlagsdienstleistungen und deren rapide steigenden Kosten (dazu unten VII./VII.) treten stärker ins Bewusstsein.

Immerhin ergibt sich eine weitgehende Bestätigung der vierten These, dass deutsche RechtswissenschaftlerInnen bislang kaum mit Hilfstätigkeiten im Publikationswesen behelligt werden, die über ihre eigentliche wissenschaftliche Tätigkeit hinausgehen. Ein Leidensdruck, der mit demjenigen von NaturwissenschaftlerInnen vergleichbar wäre, ist hier also kaum zu erwarten. Allenfalls „mit Blick auf das Prinzip des effizienten Einsatzes öffentlicher Mittel“ ${ }^{55}$ könnte die Frage nach dem komparativen Mehrwert von Papierverlagen gegenüber digitalen Infrastrukturen in Zukunft akut werden.

\section{Rechtswissenschaft ist drittmittelfern.}

Ein weiteres Argument gegen größere Verbreitung von Open Access ließe sich darin sehen, dass die Rechtswissenschaft weitgehend ohne Drittmittel auskommt, sodass die in den letzten Jahren zunehmend verstärkten Open-Access-Vorgaben von Forschungsförderern kaum Einfluss auf das rechtswissenschaftliche Publikationswesen haben sollten.

Der Schweizerische Nationalfonds (SNF) verlangt in immer verbindlicherer Form, dass die Ergebnisse der von ihm geförderten Forschung frei zugänglich sind. Im Dezember 2017 hat sich der SNF das Ziel gesetzt, dass bis zum Jahr 2020 sämtliche aus seiner Förderung hervorgehenden Publikationen Open Access veröffentlicht sein müssen. ${ }^{56}$ Auch der österreichische Fonds zur Förderung der wissenschaftlichen Forschung (FWF) „verpflichtet alle ProjektleiterInnen und ProjektmitarbeiterInnen, ihre referierten Publikationen im Internet frei zugänglich zu machen, wenn diese vollständig oder teilweise aus vom FWF geförderten Projekten

54 Engel, Journals (Fn. 50), S. 37: "Technically, there is no longer a need for publishing houses. The distribution of scientific results could be done via electronic journals made available on university servers.”; a.A. ein Vertreter der Verlagsbranche, E-Mail v. 3.5.2019: „OJS so umzugestalten, dass es mit der im deutschen Sprachraum üblichen (und m.E. gut funktionierenden) Qualitätskontrolle durch Herausgeber funktioniert, bedeutet massive Aufwendungen. Zudem kann OJS nur das Verfahren erleichtern, aber nicht die Auswahl von Gutachtern leisten. Im Moment ist die automatische Generierung von Schlagworten und anderen Metadaten noch in den Kinderschuhen. Ein Verlag, der sich nur auf automatisierte Prozesse beschränken würde, hätte massive Probleme. “.

55 Abegg, Erfahrungen (Fn. 37), S. 3: „Mit Blick auf das Prinzip des effizienten Einsatzes öffentlicher Mittel lässt es sich heute - im Zeitalter des Internets - nicht mehr rechtfertigen, den Aufwand der Wissensverteilung mit weitgehenden Nutzungsrechten zu entschädigen, damit das Recht am Zugang zu Wissen aus der (öffentlichen) Hand zu geben und folglich zu riskieren, dass die öffentlich finanzierte Wissenserarbeitung in ihrer Funktion beeinträchtigt wird.“.

56 SNF, Ab 2020: SNF-Forschung wird 100\% Open Access (perma.cc/5SWA-JK3C). 
hervorgegangen sind. "57 Demgegenüber hat die deutsche Forschungsgemeinschaft (DFG) bis heute keine verbindlichen Open-Access-Vorgaben erlassen. Die im Januar 2006 durch den Hauptausschuss der DFG verabschiedeten und in den FAQ erwähnten „Richtlinien für die Bereitstellung von Ergebnissen aus DFG-geförderten Projekten im Open Access“ sind auf der Website der DFG nicht auffindbar; dort heißt es lediglich, dass „die Sachbeihilfeempfänger der DFG ihre Forschungsergebnisse nach Möglichkeit (auch) digital und für Nutzer entgeltfrei über das Internet bereitstellen " sollen. ${ }^{58}$

Selbst diese schwach formulierten Anforderungen wären für die Rechtswissenschaft allerdings nur dann relevant, wenn Drittmittelförderung dort überhaupt gebräuchlich wäre. Daran lässt sich zweifeln, wenn etwa die Jahresstatistik des österreichischen FWF das Fördervolumen für Rechtswissenschaft 2018 auf weniger als ein Promille ihres Förderetats bezifferte, womit sie unter allen 41 geförderten Disziplinen zu den fünf drittmittelschwächsten gehörte. ${ }^{59}$ Für die vorliegende These ist indessen nicht das Fördervolumen, sondern die Anzahl der geförderten Projekte entscheidend. Diese wird nur in der Schweiz gesondert ausgewiesen, ist aber durchaus aufschlussreich. Denn während die Wirtschafts- und Rechtswissenschaften auch in der Schweiz 2018 weniger als 5 \% des bewilligten Fördervolumens erhielten, bildeten sie doch die nach Volumen und Anzahl drittmittelstärkste Disziplinengruppe innerhalb der Geistes- und Sozialwissenschaften - und kamen nach der Anzahl bewilligter Gesuche sogar auf den vierten Rangplatz aller 21 geförderten Disziplinengruppen, mit nur zwei Bewilligungen weniger als die Physik. ${ }^{60}$ Eine Suche in der Forschungsdatenbank des SNF zeigt überdies, dass in der Rechtswissenschaft mehr Projekte bewilligt werden als in der Volkswirtschafts- und der Betriebswirtschaftslehre. ${ }^{61}$ Dies belegt, dass das vergleichsweise geringe Drittmittelvolumen der deutschsprachigen Rechtswissenschaft mit einer überraschend hohen Anzahl von Drittmittelprojekten einhergeht, für die Förderrichtlinien der Forschungsförderer einschließlich darin enthaltener Open-Access-Vorgaben gelten.

Aber auch die Universitäten selbst fordern verstärkt Open Access. In Deutschland hat der baden-württembergische Gesetzgeber in $\mathbb{S} 44$ Abs. 6 des Landeshochschulgesetzes den Hochschulen seines Landes aufgegeben, „die Angehörigen ihres wis-

57 FWF, Open Access für Referierte Publikationen (perma.cc/5DXB-MA44).

58 DFG, FAQ: Open Access (perma.cc/2CJS-T8J7).

59 FWF, Jahresbericht 2018 (perma.cc/RHT9-C9BY), S. 85: im Vorjahr noch 0,5 \%; für die DFG ließ sich keine Aufschlüsselung nach Einzeldisziplinen finden.

60 SNF, Statistiken 2018, Vollversion (perma.cc/Y2LQ-QBY4), S. 8: 73 Bewilligungen; mehr hatten nur die Physik (75), die Ingenieurwissenschaften (90) und die biologischen Grundlagenwissenschaften (113).

61 Ergebnisse einer erweiterten Suche in der SNF-Forschungsdatenbank (p3.snf.ch) nach Projekten der Disziplinengruppe Wirtschaftswissenschaften und Recht: total 4.521 Projekte, davon 1.841 in den Rechtswissenschaften, 1.692 in der Volkswirtschaftslehre und 988 in der Betriebswirtschaftslehre (Stand am 17. März 2019). 
senschaftlichen Personals durch Satzung [zu] verpflichten, das Recht auf nichtkommerzielle Zweitveröffentlichung [nach $\$ 38$ Abs. 4 UrhG...] für wissenschaftliche Beiträge wahrzunehmen, die im Rahmen der Dienstaufgaben entstanden [...] sind.“ Die erste daraufhin erlassene Satzung der Universität Konstanz sieht vor, dass das wissenschaftliche Personal der Universität Konstanz wissenschaftliche Beiträge, die im Rahmen der Dienstaufgaben entstanden sind, zwölf Monate nach Erstpublikation auf dem hochschuleigenen Repositorium öffentlich zugänglich machen muss, ${ }^{62}$ sofern die Voraussetzungen nach $\mathbb{} 38$ Abs. 4 UrhG erfüllt sind. Gegen diese Satzung hat eine Gruppe von 17 ProfessorInnen der Literatur- und Rechtswissenschaften ein Normenkontrollverfahren angestrengt. ${ }^{63}$ Das Verfahren ist über Zweifeln an der Gesetzgebungskompetenz des Landesgesetzgebers einstweilen ausgesetzt, ${ }^{64}$ doch andere baden-württembergische Universitäten warten nur auf die Entscheidung und stehen bereit, ihrerseits entsprechende Satzungen zu erlassen, falls sich dies als rechtmäßig herausstellt. ${ }^{65}$

Auch in der Schweiz wird Open Access nicht nur von Drittmittelgebern, sondern vermehrt auch von den Universitäten selbst gefordert: Die Rektorenkonferenz (swissuniversities) hat im Auftrag des Staatssekretariats für Bildung, Forschung und Innovation eine nationale Open-Access-Strategie erarbeitet. Diese sieht vor, dass bis 2024 alle mit öffentlichen Geldern finanzierten wissenschaftlichen Publikationen im Internet frei zugänglich sein müssen. ${ }^{66}$ Es ist keine Ausnahme für die Rechtswissenschaft vorgesehen. Auch in Österreich strebt die Universitätenkonferenz (uniko) an, dass bis 2025 ein „Gutteil“ der wissenschaftlichen Publikationstätigkeit auf Open Access umgestellt ist. ${ }^{67}$ Das deutsche Bundesministerium für Bildung und Forschung hat im September 2016 seine Open Access-Strategie vorgelegt. Diese sieht vor, dass Open Access auch in Deutschland zum Standard des wissenschaftlichen Publizierens werden soll. ${ }^{68}$

Es zeigt sich also, dass die Rechtswissenschaft weder so drittmittelfern ist, wie man zunächst annehmen möchte, noch dass lediglich Drittmittelgeber verstärkt Open-

$62 \sqrt{ } 2$ Abs. 2 der Satzung der Universität Konstanz zur Ausübung des wissenschaftlichen Zweitveröffentlichungsrechts gemäß $\ 38$ Abs. 4 UrhG vom 10. Dezember 2015 (perma.cc/4NQV-XM92).

63 Universität Konstanz, Open Access-Satzung auf juristischem Prüfstand (perma.cc/7HQC-YCFS).

64 Universität Konstanz, Verpflichtendes Zweitveröffentlichungsrecht (perma.cc/TZE6-CF62); knappe Zusammenfassung des Prozessverlaufs bei Hamann/Graf, Wissenschaftler (Fn. 26).

65 Artikel in der Badischen Zeitung: Sollen Wissenschaftstexte im Internet frei zugänglich werden? (perma.cc/SHS2-B5W5).

66 swissuniversities, Nationale Open-Access-Strategie für die Schweiz (perma.cc/Z8X5-SW49), S. 3.

67 Arbeitsgruppe Nationale Strategie des Open Access Network Austria \& Österreichische Universitätenkonferenz, Empfehlungen für die Umsetzung von Open Access in Österreich, Version 2 (doi.org/ 10.5281/zenodo.51799); in der ersten Version vom 12. November 2015 wurde noch angestrebt, dass bis 2025 die gesamte wissenschaftliche Publikationstätigkeit in Österreich auf Open Access umgestellt ist (doi.org/10.5281/zenodo.33178).

68 BMBF, Open Access in Deutschland - Die Strategie des Bundesministeriums für Bildung und Forschung (perma.cc/VF8L-CVLW), S. 6. 
Access-Publikationen einfordern. Eine relevante Sonderstellung der Rechts- gegenüber anderen Wissenschaften lässt sich jedenfalls nicht belegen.

\section{Rechtswissenschaft ist papierbasiert und IT-fern.}

Viele JuristInnen arbeiten nach wie vor bevorzugt mit Büchern und gedruckten Zeitschriften. Wenn sie einen Aufsatz in digitaler Form erhalten, drucken sie ihn aus und lesen ihn auf Papier. Diese nicht zu unterschätzende haptisch-ästhetische Komponente könnte einer flächendeckenden Umstellung auf Open Access entgegenstehen, so die sechste These.

Dieser Einwand beruht indes auf einem verbreiteten Missverständnis: Open Access bedeutet nicht, dass ein Medium durch ein anderes ersetzt wird, sondern dass neue normative Anforderungen an die Nachnutzbarkeit von Wissenschaftserkenntnissen formuliert werden, ${ }^{69}$ die sich lediglich faktisch in einem Medium besser erfüllen lassen als im anderen. Open Access erzwingt dagegen keinen Verzicht auf Bücher oder das Lesen auf Papier. Gerade bei Dissertationen und Habilitationen wollen viele AutorInnen nach jahrelanger Arbeit ein physisches Buch in Händen halten. Das wird wohl noch lange - wenn nicht immer - so sein und das muss sich gar nicht ändern. Tatsache ist aber, dass inzwischen fast alle rechtswissenschaftlichen Fachtexte am Computer geschrieben werden und dass es eigentlich auf der Hand liegt, digital produzierte Texte medienbruchfrei auch digital zu verbreiten. Stattdessen verbringen RechtswissenschaftlerInnen Hunderte von Stunden mit dem Abtippen oder Einscannen von Texten, die an einem Computer geschrieben wurden, nur um sie wieder auf dem Computer lesen und damit arbeiten zu können.

Open Access und Papier ist kein Widerspruch (mehr). Bis vor kurzem hatten AutorInnen einer Dissertation nur die Wahl zwischen der reinen Druckpublikation in einem Verlag oder der reinen Online-Publikation ohne Verlag. Heute gibt es die Möglichkeit, Dissertationen und andere Werke sowohl gedruckt als auch frei zugänglich online zu publizieren. ${ }^{70}$ Um herauszufinden, ob Bücher weniger häufig verkauft werden, wenn sie gleichzeitig online frei verfügbar sind, hat der SNF das Projekt OAPEN-CH lanciert; dessen Resultate belegen, dass die Open-Access-Publikation keinen Einfluss auf die Anzahl der verkauften Exemplare hat. ${ }^{71}$ Dies ist mit zwei sich gegenseitig aufhebenden Effekten zu erklären: Einerseits gibt es InteressentInnen, die das physische Buch aufgrund der Online-Publikation nicht kaufen. Andererseits führt die Open-Access-Publikation zu einer viel größeren Sichtbarkeit

69 Ausf. H. Hamann, in diesem Heft, S. 102.

70 So zum Beispiel in der Buchreihe sui generis: www.sui-generis.ch/buecher.

71 SNF, OAPEN-CH - Auswirkungen von Open Access auf wissenschaftliche Monographien in der Schweiz, Bern 2018 (perma.cc/K9R3-CGXL), S. 7: "Open Access hatte statistisch gesehen keinen negativen Einfluss auf die Verkaufszahlen der gedruckten Bücher.”; näher D. Krämer, in diesem Heft, S. $205 \mathrm{ff}$. 
und damit zu einem starken Werbeeffekt. Gerade weil JuristInnen auch heute noch gerne mit Papier arbeiten, sind sie bereit, für ein Buch zu bezahlen, dessen Inhalt ihnen bereits in digitaler Form vorliegt.

Fragen der Mediennutzung sind nicht zuletzt auch Generationenfragen. Zwar gibt es bereits unter älteren JuristInnen einige, die sich sehr für die digitale Verfügbarkeit juristischer Inhalte einsetzen. Dieser Anteil steigt in den jüngeren Generationen indessen nochmals erheblich an. Besonders gut wird das beim Lesen schweizerischer Bachelor- und Masterarbeiten sichtbar: Frei zugängliche Quellen werden direkt verlinkt, während Bücher und Zeitschriften, die es nur in Papierform gibt, schlicht ignoriert werden. Selbstverständlich erwarten die Bewertenden auch heute noch, dass Studierende auch Literatur berücksichtigen, die nicht digital verfügbar ist. Sie tun es aber zunehmend seltener und die Entwicklung geht wohl dahin, dass man es ihnen in fünf oder zehn Jahren kaum noch vorwerfen kann.

Auch der Anteil von Periodika, die digital gar nicht erhältlich sind, sinkt kontinuierlich. In Deutschland sind nur noch wenige ernsthafte Wissenschaftszeitschriften rein papiergebunden, in der Schweiz ist die letzte - die Zeitschrift für Schweizerisches Recht - vor zwei Jahren dazu übergegangen, auch eine digitale Version anzubieten. Aktuell läuft die Retrodigitalisierung und bald werden sämtliche Juristentagsreferate seit 1862 (ZSR-Halbband 2) offen zugänglich sein. ${ }^{72}$ Zudem entstehen ganz neue Publikationsformate ausschließlich im Internet: Juristische Blogs ${ }^{73}$ gehören in der Schweiz bereits zum Kanon der in der Standard-Ausbildungsliteratur erörterten Publikationsformate. ${ }^{74}$ Selbst wenn also Papierbasiertheit ein entscheidendes oder auch nur überzeugendes Argument gegen Open Access wäre, so wäre es damit bald nicht mehr weit her.

\section{Verlage müssen marktgerechte Preise setzen}

Oben (zu These 4) wurde bereits der „Leidensdruck“ angesprochen, der auf der Angebotsseite des Publikationsmarktes die Frage nach dem Mehrwert von Verlagen für die Autoren ins Bewusstsein gerufen hat. Mit diesen Zweifeln an der Wertschöpfung auf Angebotsseite korrespondiert auf der Nachfrageseite in vielen Disziplinen ein ähnlicher Leidensdruck angesichts massiv gestiegener Abonnementkosten für die Nutzer der Publikationen internationaler Verlagskonzerne. ${ }^{75}$ Ist in der Rechtswissenschaft hingegen der Grad an wettbewerbsbeschränkender Konzernie-

72 Schreiben des Präsidenten des schweizerischen Juristenvereins an die Vereinsmitglieder (perma.cc/ CH4C-KU53).

$73 \mathrm{Zu}$ bisher existierenden Blogs allgemein vgl. H. Hamann, in diesem Heft, S. 88 Fn. 9; zum Völkerrechtsblog insbesondere ausf. R. Kunz, in diesem Heft, S. 186.

74 D. Hürlimann, in P. Forstmoser / R. Ogorek / B. Schindler, Juristisches Arbeiten, Eine Anleitung für Studierende, 6. Auflage, Zürich 2018, S. 184 (perma.cc/8V6C-5RRD).

75 Hamann, Freier Zugang (Fn.10), S. 104 l.Sp. (Rn. 25): ,aggressive Verkaufsstrategien und steigende Abonnementpreise bei gleichzeitig sinkenden Qualitäts- und Sorgfaltsstandards machten die Ver- 
rung geringer, so die siebte These, sollten Verlage eher gezwungen sein, ihre Preissetzung am Wettbewerb auszurichten und ihre Profitmargen zu reduzieren.

In der deutschen Rechtswissenschaft sind immerhin fast alle Wissenschaftsverlage nach wie vor kapitalmarktfern, familiengeführt und erreichen nicht annähernd die Dimensionen naturwissenschaftlicher Großverlage: Wo etwa der internationale Springer-Verlag fast 3.000 Zeitschriften und einhundert Mal so viele Bücher im Angebot hat, ${ }^{76}$ kommt unter deutschen Rechtsverlagen selbst C.H. Beck in der Sparte „Recht, Steuern, Wirtschaft“ auf nicht mehr als 78 Zeitschriften und 9.000 lieferbare Buchtitel. ${ }^{77}$ Das spricht dafür, dass juristische Wissenschaftsverlage weder vergleichbare Kostensenkungen aufgrund von Skaleneffekten und internationalem Outsourcing erreichen können, noch hinreichende Marktmacht haben, um Bibliotheken beliebige Preissteigerungen zu diktieren. Der Wettbewerb zwischen juristischen Verlagen wird denn auch als Grund dafür genannt, dass Buch- und Zeitschriftenpreise in der Rechtwissenschaft bislang „als moderat“ gelten. ${ }^{78}$

Diese Annahme eines preisdämpfenden Wettbewerbs beruht allerdings auf zwei Prämissen: Zum einen, dass die Marktgegenseite überhaupt preissensibel reagiert, also Preissteigerungen bemerkt und ihre Nachfrage anpasst. Zum anderen, dass sie eine Ausweichmöglichkeit auf Ersatzprodukte hat. Beide Annahmen erscheinen zweifelhaft:

Die Preissensibilität der Marktgegenseite (also der AbonnentInnen wissenschaftlicher Fachliteratur) wird durch eine Beobachtung in Zweifel gezogen, die soweit ersichtlich noch nie thematisiert wurde: Den kontinuierlichen Anstieg von Versandkosten für rechtswissenschaftliche Zeitschriften. Berechnete etwa der Verlag einer gewissen wirtschaftsrechtlichen Fachzeitschrift für ein Jahresabonnement 2002 noch einen Versandkostenanteil von 8,20 €, belief sich derselbe Posten im Jahr

lagstätigkeit zum profitabelsten Geschäft der Welt, mit Gewinnmargen bis zu $40 \%$. Diese Symptome prägen bis heute den Kampf der Open-Access-Verfechter gegen die betreffenden Grossverlage."; ähnl. Hamann, Open Access (Fn. 10), S. 1142 r. Sp.; jeweils m.Verw. auf R. van Noorden, The True Costs of Science Publishing: Cheap open-access journals raise questions about the value publishers add for their money, Nature 495 (2013), S. 426.

76 Vgl. www.springer.com, abgerufen am 21.1.2019: “Our business is publishing. With more than 2,900 journals and 290,000 books, Springer offers many opportunities for authors, customers and partners".

77 Vgl. rsw.beck.de/verlag, abgerufen am 21.1.2019: „mehr als 9.000 lieferbare Werke [... und] über 70 Fachzeitschriften “; genaue Zeitschriftenzahl nach eigener Auszählung der unter rsw.beck.de/cms/ OverviewPage/Magazines gelisteten Titel, darunter aber auch drei GRUR-Ableger (GRUR-Int, GRUR-Prax und GRUR-RR) und vier weitere „Rechtsprechungs-Reports“ (NJW-RR, NStZ-RR, NVwZ-RR und NZA-RR). Nicht berücksichtigt sind dagegen Auslandsbeteiligungen (etwa an den Schweizer Verlagen Helbing und Dike), die zusätzliche Marktmacht vermitteln.

78 So Verch/Wiesner, Der freie Zugang zu wissenschaftlicher Information: Rechtswissenschaft, Stand 2018, abgerufen am 21.1.2019 unter perma.cc/Z8YL-ZRZM: „Da in vielen Arbeitsbereichen aktuelle juristische Literatur benötigt wird und die Nachfrage dementsprechend hoch ist, sind die Buchund Zeitschriftenpreise gerade im Vergleich zu naturwissenschaftlichen Fächern als moderat zu bezeichnen. Auf dem juristischen Publikationsmarkt in Deutschland sind zahlreiche Wettbewerber aktiv“. 
2018 bereits auf 40,00 €. Das entspricht einer Steigerung von $488 \%$ im selben Zeitraum, in dem das Briefporto der Deutschen Post (DPAG) um gerade einmal $25 \%$ gestiegen ist. ${ }^{79} \mathrm{Da}$ sich weder der Erscheinungsrhythmus noch der Umfang der Zeitschrift nennenswert geändert haben, ist der Anstieg wohl kaum anders zu erklären als durch freihändiges Abschöpfen einer Zahlungsbereitschaft der Marktgegenseite, die gerade nicht preissensibel reagiert oder reagieren kann. ${ }^{80}$ Die folgende Grafik illustriert diesen Befund für zehn Fachzeitschriften, deren Versandkostenanteile 2002-2018 öffentlich dokumentiert sind, ${ }^{81}$ ohne dass die einzelnen Verlaufslinien individuell bezeichnet werden bräuchten: Ganze sechs der zehn Zeitschriften haben ihre Versandkosten mehr als doppelt so schnell erhöht wie die Post das Briefporto.

Abb. 1: Preisindex 2002-2018 für Versandkosten zehn juristischer Fachzeitschriften. Jede gepunktete Linie stellt eine Zeitschrift dar, die Werte sind normalisiert auf Prozent des Anfangsjahres der Zeitreihe (Jahr $2002=100 \%$ ). Als durchgehende Linie abgetragen ist die Entwicklung des DPAG-Portos für einen einfachen Brief.

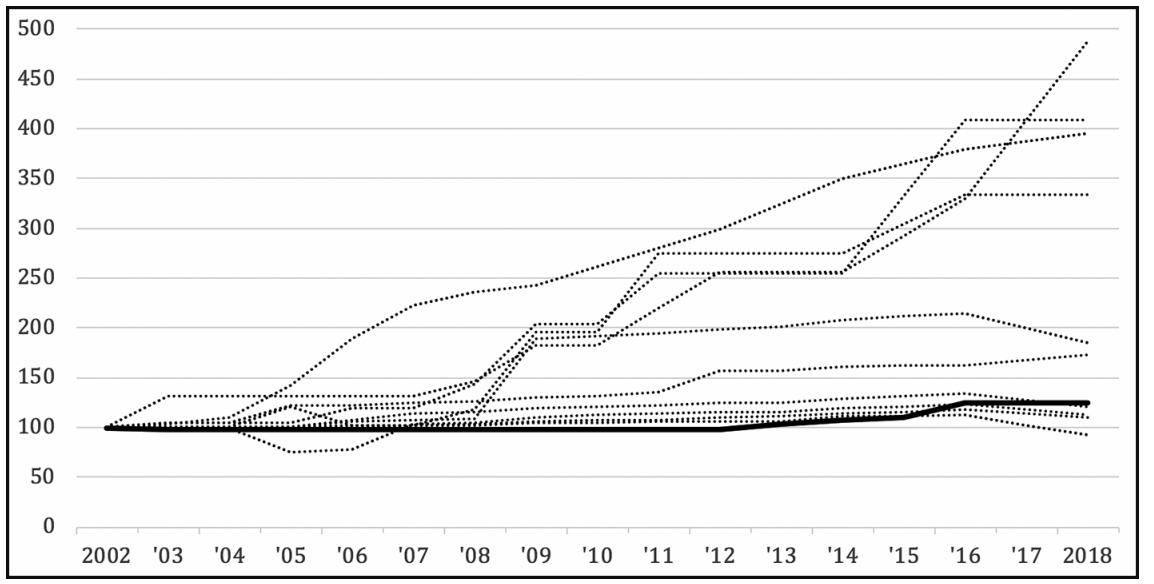

79 Zeitschriften werden zwar nicht als Brief versandt, und aus der Verlagsbranche wurden wir darauf hingewiesen (Mail v. 3.5.2019), dass „für den Versand von Zeitschriften [...] völlig andere Preise“ gelten; unsere Nachfrage nach einer Konkretisierung blieb allerdings unbeantwortet, und nach Auskunft des Posthistorikers Jürgen Olschimke (Telefonat v. 15.8.2019) veröffentlicht die Post seit etwa 2000 keine Preise mehr für ihre Produkte „Post- bzw. Pressevertriebsstück“ oder „Streifbandzeitung “ - belastbare Daten seien deshalb kaum zu finden, aber die Entwicklung dürfte „so ungefähr“ dem Briefporto entsprechen. (Wofür auch die Tatsache spricht, dass die Versandkosten für vier der zehn betrachteten Zeitschriften in Abb. 1 ungefähr dem Briefporto folgen.) Wir nehmen deshalb an, dass der gewerbliche Versand jedenfalls keinen schnelleren Preisanstieg erfahren hat als das Briefporto, und verwenden dessen Entwicklung als Indikation für die Versandkostenentwicklung insgesamt.

80 Dazu schon Engel, Journals (Fn. 50), S. 36: "at the user level, cost played no role in the decision of individual researchers to access material. [...] The commercial base and the user level were thus separate.”.

81 Daten für ARSP, DÖV, JR, JuS, NJW, NVwZ, ZEuP, ZGR, ZRP und ZStW aus dem jährlichen Verzeichnis „Banger Zeitschriften“ (Köln 2002-2016), seit 2017 von den jeweiligen Verlagswebsites. 
Auch die zweite Annahme, dass AbonnentInnen eine Ausweichmöglichkeit auf Substitute zur Verfügung steht, erscheint bei wissenschaftlichen Fachpublikationen fraglich. Zwar verfügt die deutschsprachige Rechtswissenschaft immerhin noch über zwei Dutzend größerer Fachverlage, ${ }^{82}$ auch wenn einige davon ausschließlich Nischensegmente bedienen und in den Hauptrechtsgebieten kaum konkurrieren. Für die Ausweichmöglichkeiten der Marktgegenseite kommt es indessen ökonomisch nicht auf die Substituierbarkeit der Anbieter, sondern diejenige der Produkte an. ${ }^{83}$

Dabei sieht man schnell, dass jede Fachzeitschrift (genaugenommen sogar jeder einzelne Artikel) ein Monopolprodukt darstellt, weil nach traditionellem Verlagsrecht $(\$ 9$ II VerlG) jeweils nur ein Verlag diesen Inhalt liefern kann. Juristische Fachbibliotheken können sich also im Strafrecht nicht zwischen ZStW (de Gruyter) und GA (C.F. Müller) entscheiden, im Zivilrecht nicht zwischen AcP (Mohr Siebeck) und ZfPW (C.H. Beck) und im Gesellschaftsrecht nicht zwischen ZHR (dfv Mediengruppe) und ZGR (de Gruyter), sondern benötigen in jedem Rechtsgebiet die Inhalte jeweils beider Zeitschriften - zumindest solange diese genug Ansehen genießen, um von Universitätsangehörigen nachgefragt zu werden, die mit der Preisentwicklung tendenziell nicht vertraut (also wiederum nicht preissensibel) sind. Mithin konkurrieren die Verlage solcher Zeitschriften effektiv letztlich weniger um AbonnentInnen als allenfalls um AutorInnen. ${ }^{84}$

Daraus wiederum folgt, dass ein nennenswerter Preiswettbewerb unter juristischen Fachverlagen jedenfalls nicht in der Marktstruktur angelegt ist. Dass er eine Gewähr für „moderate“ Preise böte, lässt sich deshalb nicht ohne Weiteres annehmen. Vielmehr können Verlage quasi-Monopolgewinne abschöpfen (rent extraction), die in einem gewöhnlichen Wettbewerbsumfeld so nicht zu erzielen wären. ${ }^{85}$

\section{Juristische Verlagspreise sind angemessen.}

Die bloße Macht zur Monopolausbeutung bedeutet freilich noch nicht, dass Verlage sie auch nutzen. Immerhin gilt gerade die Rechtswissenschaft als Hort gegenseitigen Vertrauens und gelingender informeller Verständigung zwischen AutorInnen und VerlegerInnen, ${ }^{86}$ sodass der andernorts beklagte Wechsel von Verlagen „aus

82 Mitgliederliste der Arbeitsgemeinschaft der rechts- und staatswissenschaftlichen Verlage e.V., abgerufen am 21.1.2019 unter www.juristische-verlage.de.

83 Engel, Journals (Fn. 50), S. 35: "In antitrust terminology: What is the relevant product market?".

84 Engel, Journals (Fn. 50), S. 36: "Vis-à-vis the reader, each individual article is a monopoly product. Articles are, at most, remote substitutes. [...] Vis-à-vis the author, the proximity of substitutes rests on the vocation of the journal, and on its ranking.".

85 Engel, Journals (Fn. 50), S. 36: "for a mere distribution service, the publishing houses get the property right to the contents. [...] This excessive property right generates market power."; ähnl. Abegg, zit. in Fn. 37.

86 Stellvertretend nur I. v. Münch / G. Siebeck, Der Autor und sein Verlag, Tübingen 2013. 
der Rolle des Mehrwert-Dienstleisters immer öfter in die des Mehrwert-Ausbeuters" ${ }^{87}$ völlig ausgeblieben sein könnte. Dann entfiele, so unsere letzte These, womöglich auch das Kostenargument zugunsten Open Access.

Inwiefern freilich juristische Verlagspreise „moderat“ oder „angemessen“ ausfallen, lässt sich nur mithilfe von Vergleichsdaten erkennen. Deshalb vergleichen USamerikanische Bibliotheken bisweilen die Preisentwicklung wissenschaftlicher Zeitschriften mit der Inflation im gleichen Zeitraum. Dadurch lassen sich „übermäßige“ Renditesteigerungen identifizieren, die sich durch die allgemeine Teuerung nicht erklären lassen. Entsprechend grafisch dargestellte Zeitreihen für 1986-2006 und 2007-2013 lassen einen drei- bis viermal so schnellen Anstieg von Zeitschriftenpreisen gegenüber der Inflation erkennen. ${ }^{88}$

Diese Erhebungen aus dem US-amerikanischen Umfeld lassen sich freilich nicht direkt auf die deutsche Rechtswissenschaft übertragen. Die US-amerikanische Literaturversorgung unterscheidet sich so gravierend vom deutschen Marktumfeld allgemein und vom juristischen Publikationsmarkt insbesondere, ${ }^{89}$ dass die Frage nach den hiesigen Preissteigerungen einer eigenen Erhebung bedarf..$^{90}$

Für diese Erhebung wurden 35 rechtswissenschaftliche Fachzeitschriften ausgewählt, die alle renommierten Archiv-, Ausbildungs- und anderen rechtsgebietsübergreifenden Zeitschriften umfassten, sowie wichtige Zeitschriften dreier ReferenzRechtsgebiete - wie aus folgender Tabelle ersichtlich:

Tab. 1: 35 rechtswissenschaftliche Fachzeitschriften aus Deutschland, deren Abonnementpreise für die vorliegende Studie ausgewertet wurden.

\begin{tabular}{lcl}
\hline Kategorie & Anz. & Zeitschriftenkürzel \\
\hline Archivzeitschriften & 10 & ARSP, AcP, AfP, AöR, GA, RT, Staat, ZG, ZStW, ZZP \\
\hline Ausbildungszeitschriften & 3 & JA, JURA, JuS \\
\hline rechtsgebietsübergreifend & 9 & JR, JZ, KJ, KritV, MDR, NJW, VuR, ZfRSoz, ZRP \\
\hline Verwaltungsrecht & 5 & DVBl, DÖV, NVwZ, Verw., VerwArch \\
\hline Rechtsvergleichung & 4 & RabelsZ, ZEuP, ZVglRWiss, ZaöRV \\
\hline Wirtschaftsrecht & 4 & RIW, ZGR, ZHR, ZIP \\
\hline
\end{tabular}

87 Hamann, Open Access (Fn. 10), S. 1142 r. Sp.; ders., Freier Zugang (Fn. 10), S. 104 l.Sp. (Rn. 25).

88 Bspw. Western Illinois University, Verlaufsgrafik "Trends in Book and Journal Spending in ARL Libraries, 1986-2006", abgerufen am 21.1.2019 von wiu.libguides.com/c.php? $\mathrm{g}=295451 \& \mathrm{p}=1969198$; UC Santa Barbara Library, Verlaufsgrafik "Price Inflation of Academic Books and Journals Compared to Overall US Inflation, 2007-2013”, abgerufen 21.1.2019 von web.archive.org/20171222232622/library.ucsb.edu/collection-development/budget-challenges.

89 Dazu oben nach Fn. 37 und zuvor schon in Hamann, Wissenschaftsverlag (Fn. 32), S. 32 („Die juristische Verlagslandschaft").

90 Zuvor für die Schweiz bereits Dengg, Infrastrukturen (Fn. 8), dazu gleich Fn. 95. 
Diese 35 Zeitschriften stehen für 12 verschiedene Verlage, die je 1-5 Zeitschriften zur Stichprobe beisteuern (Median 2 / ø 2,3). ${ }^{91}$ Für jede Zeitschrift wurde der von ihrem Verlag veröffentlichte Grundpreis eines institutionellen Abonnements in jedem Jahr seit Einführung des Euro (2002) erhoben und wie schon oben bei den Versandkosten in Prozent des Wertes von 2002 skaliert. $^{92}$ Dabei wurde bewusst hingenommen, dass (wie von einzelnen Verlagsvertretern in informellen Gesprächen angemerkt) die annoncierten Preise womöglich nicht den realen entsprechen, da kein Verlag bisher bereit war, seine realen Abonnementpreise offenzulegen.

Trägt man die vom Bundesamt für Statistik dokumentierte Teuerungsrate zu Jahresbeginn (Inflation) ab, ergibt sich beispielhaft die folgende Zeitreihe. Sie zeigt neben dem vom Bundesamt genutzten Inflationsmaß „Verbraucherpreisindex“ die Preisentwicklung der hier untersuchten Zeitschriften des Kohlhammer-Verlags; da der Kohlhammer-Verlag nur mit einer einzigen Zeitschrift vertreten ist (DÖV), werden die Datenpunkte ausgehöhlt dargestellt.

Abb. 2: Preisindex 2002-2018 für das Jahresabonnement der Zeitschrift DÖV (O) und den amtlichen Verbraucherpreisindex $(x)$. Alle Werte sind normalisiert auf Prozent des Anfangsjahres der Zeitreihe (Jahr $2002=100 \%)$.

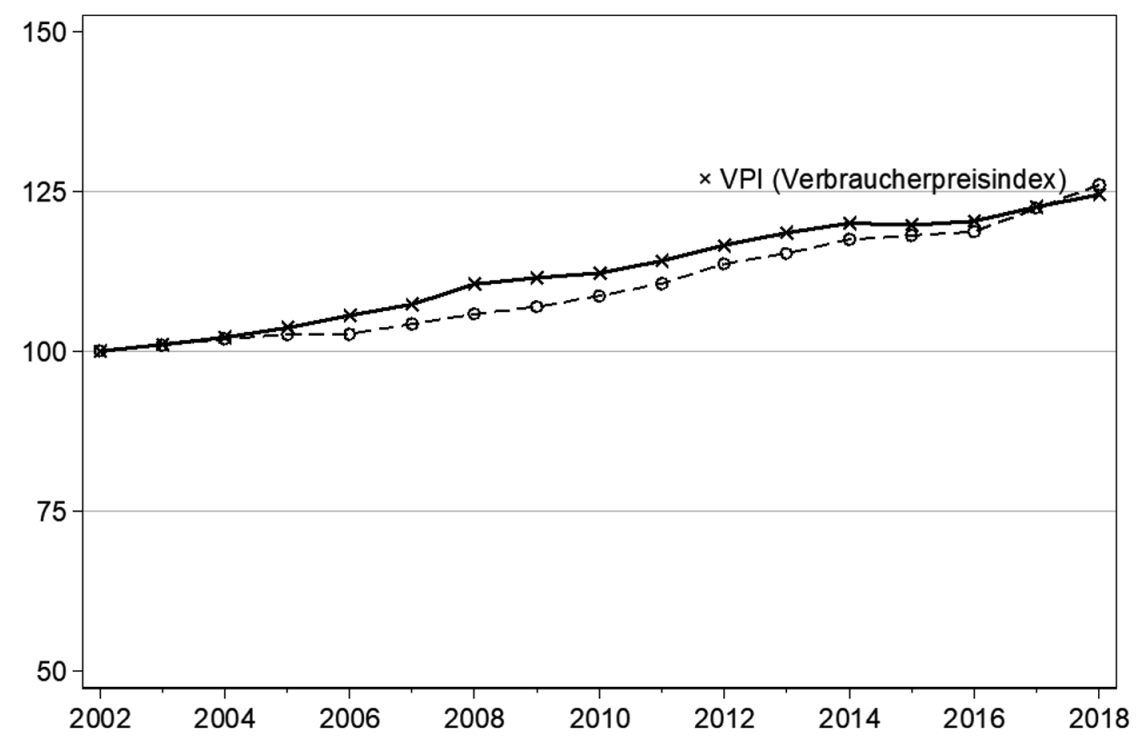

Die dargestellte Preisentwicklung der DÖV kompensiert folglich recht genau den Inflationsanstieg und sichert damit dem Kohlhammer-Verlag seine Profitmarge des

91 Nach absteigender Häufigkeit: C.H. Beck (5), de Gruyter, Mohr Siebeck (je 4), Heymanns, Duncker, R\&W (je 3), Nomos, Kohlhammer, Luchterhand, Hüthig (je 2), sowie Lucius \& Lucius, RWS, Franz Steiner, Handelsblatt und O. Schmidt (je 1).

92 Datenquelle wie oben in Fn. 81. 
Jahres 2002 dagegen, durch die allgemeine Teuerung von zwischenzeitlich 24,5\% aufgezehrt zu werden. Freilich erscheint der Verbraucherpreisindex (VPI) nicht als besonders geeignete Vergleichsmetrik, da Zeitschriften als gewerblich hergestellte Industrieprodukte eigenen Inflationsdynamiken unterliegen. Ersetzt man diesen Index deshalb durch Bruttolöhne im Verlagswesen (Arbeitskostenindex J58), ändert sich die Trendlinie allerdings kaum: Die Teuerung seit 2002 betrug hier 24,6\%. Der Materialpreis für den Druck von Zeitschriften dagegen (Erzeugerpreisindex \#160) ist seit 2002 sogar kontinuierlich gefallen, um immerhin insgesamt 21,8\%. Da sich die Gesamtkosten eines Verlags im Wesentlichen aus diesen beiden Produktionsfaktoren zusammensetzen, ist anzunehmen, dass die effektive Teuerungsrate der Verlagstätigkeit seit 2002 zwischen diesen beiden Werten (Lohnkosten $+24,6 \%$, Materialkosten $-21,8 \%$ ) liegen dürfte. Das Bild jedoch, das sich zeigt, wenn man neben der Zeitschrift des Kohlhammer-Verlags die übrigen elf Verlage abträgt,${ }^{93}$ liegt erheblich außerhalb dieser Spanne:

Abb. 3: Preisindex 2002-2018 normalisiert $(2002=100 \%)$ für das Jahresabonnement von 35 juristischen Fachzeitschriften. Jede gestrichelte Linie bildet einen von zwölf Verlagen ab (ausgehöhlte Datenpunkte je 1 Zeitschrift), die durchgezogenen Linien die beiden herangezogenen Vergleichsindizes.

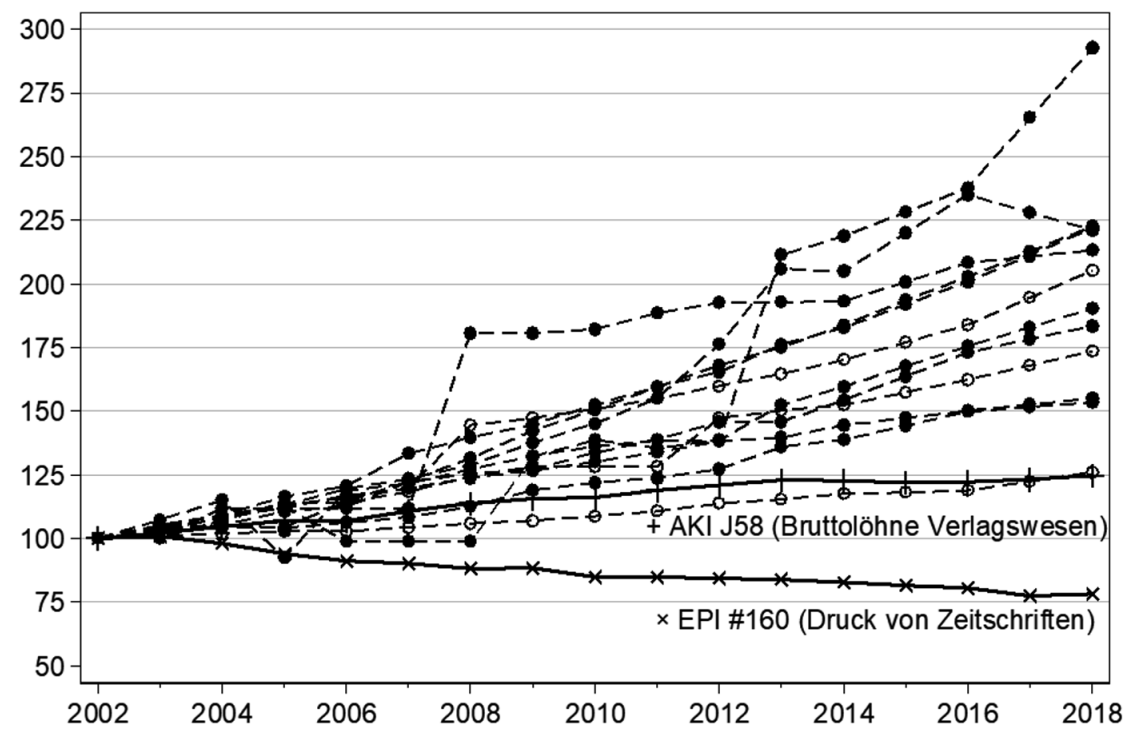

Die Abbildung zeigt, dass nur die Preispolitik des Kohlhammer-Verlags überhaupt in der Nähe der durch Inflation begründbaren Preissteigerungen liegt. Andere Ver-

93 Weitere Verlage und Zeitschriften werden nicht namhaft gemacht, weil die Erhebung nicht dazu dient, einzelne Verlage anzuprangern, sondern systematische Zusammenhänge zu untersuchen. Kohlhammer und die DÖV wurden nur zur Illustration benannt, weil es die Erläuterung des Diagramms erleichtert und keine negative Deutungsmöglichkeit dieses Befunds ersichtlich ist. 
lage haben ihre Zeitschriftenpreise (meist sogar über mehrere Zeitschriften hinweg) verdoppelt, einer sogar verdreifacht. Damit liegt die Preissteigerung im selben Bereich wie er für die Schweiz bereits dokumentiert wurde. ${ }^{94}$

Diesen Befund mag man zunächst mit einer anderen Tendenz im Verlagswesen zu erklären versuchen: Mit sinkenden Verkaufszahlen. Soweit Zeitschriften seit 2002 LeserInnen eingebüßt hätten (und keine Vergrößerung der Leserschaft durch Qualitätssteigerung möglich wäre) würde die Sicherung ihrer Rentabilität natürlich eine Preissteigerung erfordern. Doch erhebt man zusätzlich zu den Preis- auch die Auflagenzahlen, ${ }^{95}$ belegen die Daten keineswegs deren generellen Verfall:

Abb. 4: Auflagenindex 2002-2018 normalisiert (2002 = 100 \%) für die jährliche Nominalauflage von 32 juristischen Fachzeitschriften. ${ }^{96}$ Jede Linie bildet eine Zeitschrift in den Jahren 2002, 2010 und 2018 ab. Ausgehöhlte Datenpunkte (O) markieren Auflagenverluste, gefüllte Datenpunkte (匹) Auflagenzuwächse.

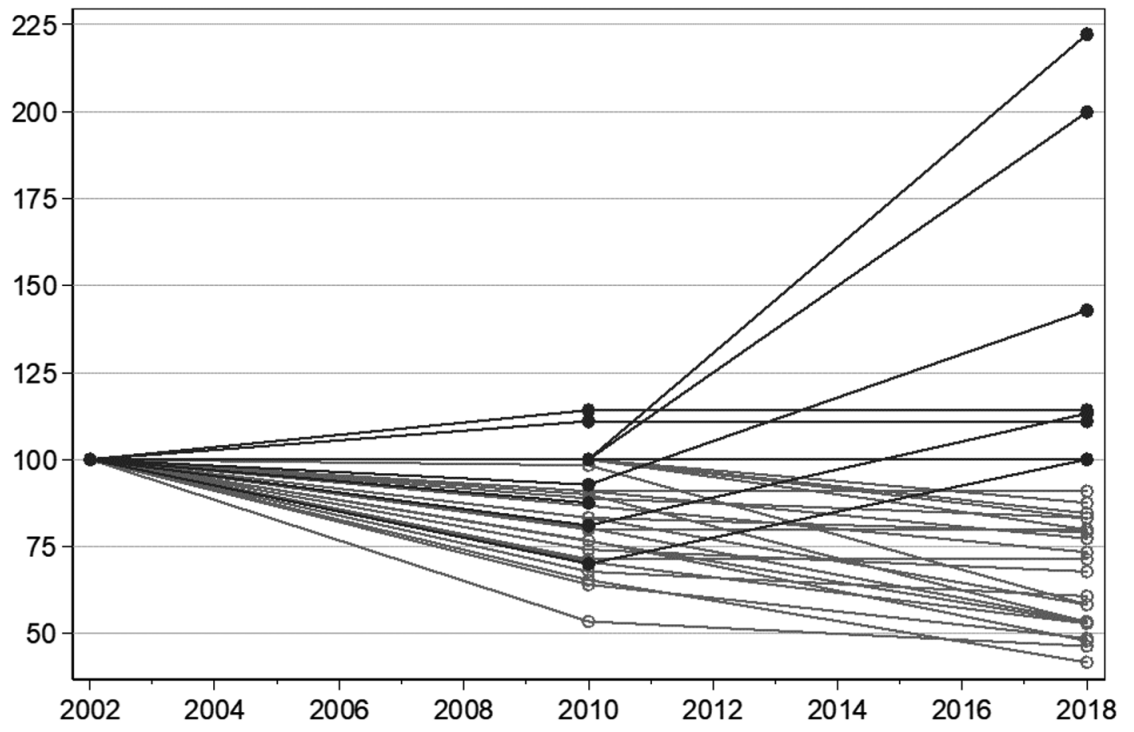

94 Eigene Auswertung der Daten aus Dengg, Infrastrukturen (Fn. 8), S. 26: Steigerung der Zeitschriftenkosten an der Juristischen Bibliothek Bern von $41 \%$ über alle Rechtszeitschriften hinweg im Zeitraum 2005-2015.

95 Daten aus dem jährlichen Verzeichnis „STAMM Leitfaden durch Presse und Werbung“ (Essen 2002-2018), Sachgruppen 32790 („Staats- und Rechtswissenschaften“), 32410 („Verwaltung allgemein“: DÖV, VerwArch), 32500 („Steuer-, wirtschafts- und rechtsberatende Berufe“: JA, JuS), 32660 („Philosophie und Psychologie“: ARSP). Dem Einwand aus der Verlagsbranche (Mail v. 3.5.2019), „dass die dort genannten Auflagen nicht immer zuverlässig sind und die real verkauften Abos wiedergeben " lässt sich nur entgegenhalten, dass der Leitfaden lediglich dokumentiert, was Verlage selbst öffentlich angeben; zur (selten) gesondert ausgewiesenen Effektivauflage vgl. noch Fn. 98 a.E.

96 Für drei Zeitschriften lagen keine lückenlosen Daten vor: VuR, ZaöRV und ZRP. 
Die Darstellung zeigt, dass zwar 24 der 35 untersuchten Zeitschriften an Auflagenstärke verloren haben - bis zu knapp $60 \%$ ihrer Nominalauflage ${ }^{97}$-, acht Zeitschriften hingegen Zuwächse verzeichnen und ihre Auflage zum Teil sogar mehr als verdoppeln konnten. ${ }^{98}$ In Anbetracht dieses durchaus gemischten Bildes ist nicht klar, warum die zuvor festgestellte Preissteigerung für alle Verlage erforderlich gewesen sein sollte, um einen (vermeintlichen) Verfall der Auflagenzahlen zu kompensieren. Zuletzt sei deshalb der Umsatz betrachtet, den die 12 untersuchten Verlage mit den Zeitschriften der Stichprobe erzielen konnten: Unter der vereinfachenden Annahme, dass die Nominalauflagen komplett durch institutionelle Abonnements abverkauft werden, ${ }^{99}$ lässt sich durch Multiplikation der Auflagenhöhe mit dem Abonnementpreis eine grobe Abschätzung für den Umsatz gewinnen, den ein Verlag mit seinen Zeitschriften erzielt; Quersubventionierungseffekte innerhalb eines Verlagshauses werden dadurch direkt berücksichtigt. Die absolute Höhe dieser Umsätze mag hier dahinstehen, einzig interessant ist deren Entwicklung seit 2002:

97 Um Restbestände zu berücksichtigen, weisen manche Zeitschriften auch ihre effektive Auflage aus: Bei der NJW bspw. betrug im Zeitraum 1.7.2017-30.6.2018 die Druckauflage 27.927 Exemplare, die „tatsächlich verbreitete Auflage im Jahresdurchschnitt“ dagegen nur 26.405 Exemplare (NJWMediadaten, abgerufen am 22.1.2019 von www.anzeigenbuchung.beck.de/system/files/mediadaten/n jw_mediadaten_2019.pdf ), also 5,4\% weniger. Größere Abweichungen zwischen beiden Werten wären betriebswirtschaftlich verlustträchtig und sind daher kaum zu erwarten. Da die effektive Auflage weniger gut dokumentiert ist als die nominale, wird vorliegend letztere verwendet.

98 Die drei Zeitschriften mit dem stärksten Auflagenanstieg gehören zwar alle zum selben Verlag; er war aber bei einer weiteren Zeitschrift auch von einem 26-prozentigen Auflageneinbruch betroffen.

99 Trotz Kritik aus der Verlagsbranche an dieser Vereinfachung (Mail v. 3.5.2019) wurden auch auf Nachfrage leider keine empirischen Daten zur Verfügung gestellt, aus denen sich Korrekturfaktoren hätten errechnen lassen. Das erscheint letztlich auch verzichtbar, weil (1) noch heute über zwei Drittel der Zeitschriften (24 von 35) einen einheitlichen Preis für institutionelle und individuelle Abonnements angeben und (2) lediglich Indexentwicklungen betrachtet werden, die beide Abonnementtypen ähnlich betreffen: Diejenigen Zeitschriften, die überhaupt preislich differenzieren, erhöhten über die vergangenen fünf Jahre hinweg (2013-2018) ihren institutionellen Preis um 22,2\%, den individuellen dagegen um 15,6\%. Das entspricht einem Unterschied von gerade einmal 1,3 Prozentpunkten pro Jahr, der die Trendaussagen im Haupttext kaum berührt. 
Abb. 5: Umsatzindex 2002-2018 normalisiert (2002 = 100\%) für den Nominalumsatz (Nominalauflage $x$ Jahresabonnementpreis) von elf untersuchten Verlagen ${ }^{100}$ mit ihren in der Stichprobe enthaltenen Zeitschriften. Jede Linie bildet einen Verlag in den Jahren 2002, 2010 und 2018 ab. Ausgehöhlte Datenpunkte (O) markieren Umsatzverluste, gefüllte Datenpunkte (匹) Umsatzzuwächse.

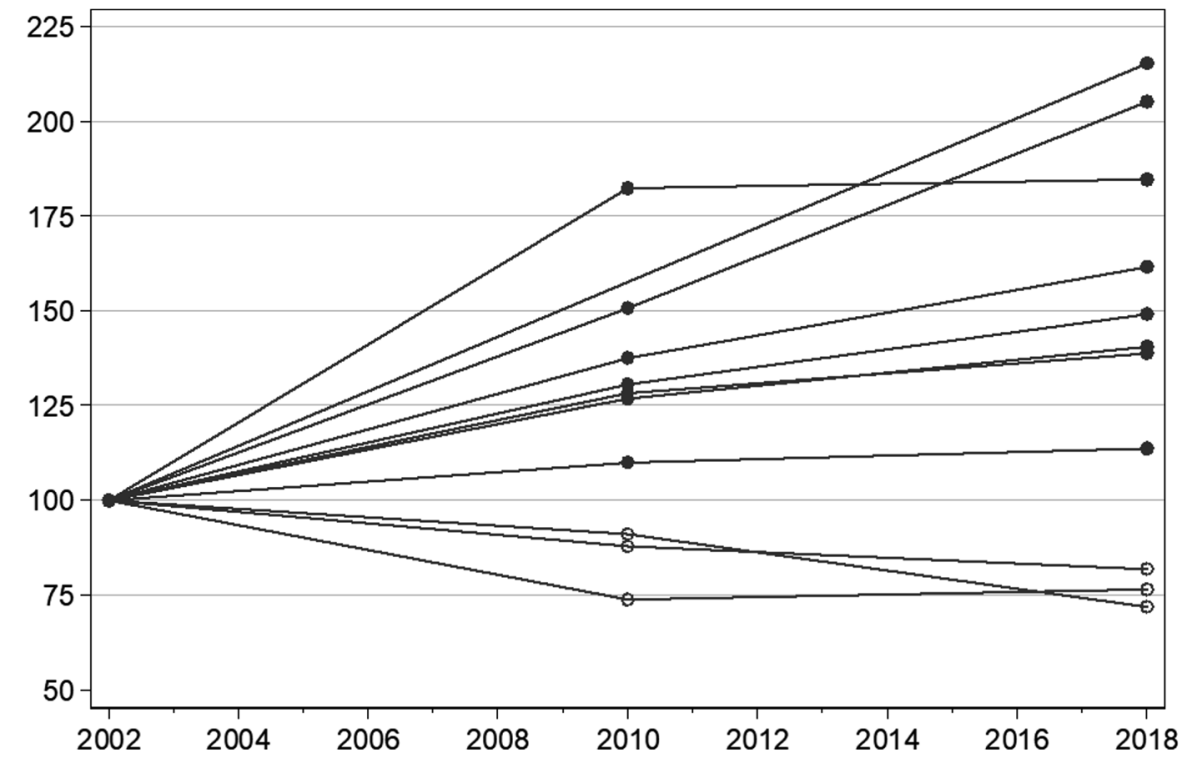

Die Abbildung zeigt also, dass nach dieser Berechnung nur drei Verlage überhaupt Umsatzeinbußen verzeichnen mussten. Die anderen acht Verlage hingegen konnten Umsatzgewinne von bis zu $115 \%$ verzeichnen; über alle Verlage hinweg betrug das mittlere Umsatzplus 39,9\% (Median 40,4\%) in nur 16 Jahren. ${ }^{101}$ Dadurch werden Umsatzrenditen möglich, die der Cheflektor eines (in der vorliegenden Auswertung vertretenen) Verlags in privater Korrespondenz auf „über 30 Prozent“ bezifferte, also durchaus vergleichbar zu den „Gewinnmargen bis zu 40 \% “ der internationalen Wissenschaftsverlage, die andere Disziplinen als anstößig empfinden. ${ }^{102}$

Die Auswertung belegt damit, dass auch für die deutsche Rechtswissenschaft eine bemerkenswert schnelle Steigerung der Zeitschriftenpreise (zusätzlich zu ebensolcher Versandkostensteigerung, s.o. Abb. 1) zu verzeichnen ist, die sich weder durch sinkende Auflagen noch durch die allgemeine Teuerung erklären lässt. Dieser Be-

100 Ein Verlag wurde vorsichtshalber aus der Abbildung entfernt, weil seine exorbitante Auflagensteigerung - vgl. Fn. 98 - zu einem nominalen Umsatzsprung auf fast $360 \%$ geführt hätte.

101 Unter Einschluss des 12. Verlags (vorige Fn.) läge das mittlere Umsatzplus sogar bei 58,2 \% (Median $44,8 \%$ ).

102 Vgl. Zitat oben Fn. 75 - damals ebd. noch mit der (zunehmend unplausiblen) Vermutung, ,ein etwas anderes Bild bieten wohl deutsche Rechtsverlage“. 
fund mag dazu anregen, auch die achte These zumindest ernsthaft in Frage zu stellen und ebenso wie in anderen Wissenschaftsdisziplinen über die Kostenvorteile eines Open-Access-Modells nachzudenken: Sobald Zeitschriften nicht mehr durch Abonnenten finanziert werden, die strukturell wenig preissensibel sind und keine Ausweichmöglichkeiten haben (s.o. zu These VII), sondern durch AutorInnen oder Forschungsförderer, dürfte sich eine größere Konkurrenz um AutorInnen einstellen, die zu einem höheren Bewusstsein für den Wert echter verlegerischer Dienstleistungen führt und dafür eine angemessene Vergütung am Markt finden hilft, statt eine - bewusst oder unbewusst - freihändige Abschöpfung von Monopolgewinnen zuzulassen.

\section{Fazit}

Nach der Sichtung und argumentativen Bearbeitung von acht Thesen, die gegen eine stärkere Durchsetzung von Open Access in der deutschsprachigen Rechtswissenschaft sprechen, bleibt ein gemischtes Bild. Einerseits enthalten alle acht Thesen einen wahren Kern, weshalb sich die Auseinandersetzung mit ihnen lohnt, um zu verstehen, warum Open Access in der Rechtswissenschaft so zögerlich aufgenommen wird. ${ }^{103}$

Andererseits hat die Auseinandersetzung mit den Thesen gezeigt, dass sich jedenfalls keine durchschlagenden Hindernisse oder besonderen institutionellen Rahmenbedingungen erkennen lassen, die unterschiedliche Open-Access-Durchsetzungsraten in Rechts- und anderen Wissenschaften schlüssig erklären könnten. Auch rechtswissenschaftliche Publikationen aus dem deutschen Sprachraum werden von Fachfremden und von Angehörigen anderer Jurisdiktionen gelesen und zitiert, wenn sie denn die Möglichkeit dazu haben. Auch Open-Access-Zeitschriften können sich ein Renommee erarbeiten, das für Karrierewege förderlich ist. Dazu tragen auch die Vorgaben der Drittmittelgeber bei, die nicht selten juristische Forschungsprojekte fördern und dabei verlangen, dass die Ergebnisse Open Access publiziert werden. Bücher werden in der Rechtswissenschaft weiterhin eine zentrale Rolle spielen, sie werden auch weiterhin gedruckt, was der gleichzeitigen OpenAccess-Publikation aber nicht im Wege steht. Während in anderen Disziplinen massive Preissteigerungen die Umstellung auf Open Access beschleunigt haben, lassen sie sich - bislang ohne vergleichbaren Aufruhr - auch in der Rechtswissenschaft bereits beobachten. Schon heute müssen viele Bibliotheken mit Blick auf ihr Budget entscheiden, welche Zeitschriften und Bücher sie sich noch leisten können. Aus diesem Grund sind auch sie an einer Umstellung auf Open Access interessiert.

Die im Titel des Beitrags gestellte Frage kann also der folgenden Antwort zugeführt werden: Open Access wird mittelfristig auch in der Rechtswissenschaft zum 
Standard werden, weil diese Art des Publizierens die Reichweite erhöht, den raschen Austausch mit anderen Disziplinen sowie Jurisdiktionen fördert und nicht zuletzt von öffentlichen Geldgebern - ob Forschungsförderer oder Universitäten erwartet wird. Institutionelle Eigenheiten der Rechtswissenschaft wie ihre bislang zögerliche Metrisierung, ihre Papierbasiertheit und ihr Verzicht auf Peer Review haben zur festgestellten Verzögerung der Umstellung auf Open Access geführt, werden diese aber mittelfristig nicht verhindern können. 